\title{
Occupational exposure to electromagnetic fields in magnetic resonance environment: an update on regulation, exposure assessment techniques, health risk evaluation and surveillance.
}

Valentina Hartwig ${ }^{1, *}$, Giorgio Virgili ${ }^{2}$, Federica Mattei $^{3}$, Cristiano Biagini ${ }^{4}$, Stefania Romeo ${ }^{5}$, Olga

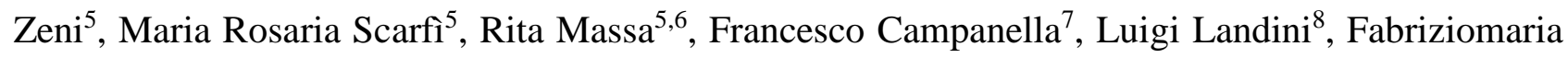
Gobba $^{9}$, Alberto Modenese ${ }^{9}$, Giulio Giovannetti ${ }^{1}$

${ }^{1}$ Italian National Research Council (CNR), Institute of Clinical Physiology (IFC), Via G. Moruzzi 1, 56124 San Cataldo, Pisa, Italy

${ }^{2}$ Virgili Giorgio, Via G. Pastore 2, 26040, Crespina-Lorenzana, Italy

${ }^{3}$ West Systems S.r.l., Via Don Mazzolari 25, 56025, Pontedera (PI), Italy

${ }^{4}$ Associazione Italiana Tecnici dell'Imaging in Risonanza Magnetica, AITIRM, Via XX Settembre 76, 50129, Firenze, Italy

${ }^{5}$ Italian National Research Council (CNR), Institute for Electromagnetic Sensing of the Environment (IREA), Via Diocleziano 328, 80124 Naples, Italy

${ }^{6}$ Department of Physics, University Federico II, Via Cinthia 21, 80126 Napoli, Italy

${ }^{7}$ Inail, Dipartimento di medicina, epidemiologia, igiene del lavoro e ambientale, Via Fontana Candida 1, 00078 Monte Porzio Catone, Roma, Italy

${ }^{8}$ Fondazione CNR-Regione Toscana “G. Monasterio’, Via G. Moruzzi 1, 56124, San Cataldo, Pisa, Italy

${ }^{9}$ Department of Biomedical, Metabolic and Neural Sciences, University of Modena and Reggio Emilia, Via Campi 287, 41125 Modena, Italy

*Eng. V. Hartwig, PhD

Institute of Clinical Physiology - CNR

Via Moruzzi 1, 56124 S. Cataldo, Pisa, Italy.

E-mail: valeh@ifc.cnr.it

Phone: +390503152827

Total number of words of the manuscript, including entire text from title page to figure legends: 12938

Number of words of the abstract: 143

Number of figures: 2

Number of tables: 1 


\section{Abstract}

Magnetic Resonance Imaging (MRI) is one of the most-used diagnostic imaging methods worldwide. There are 50,000 MRI scanners worldwide each of which involves a minimum of five workers from different disciplines who spend their working days around MRI scanners. This review analyses the state-of-art of literature about the several aspects of the occupational exposure to electromagnetic fields (EMF) in MRI: regulations, literature studies on biological effects, and health surveillance are addressed here in detail, along with a summary of the main approaches for exposure assessment. The original research papers published from 2013 to 2021 in international peer-reviewed journals, in the English language, are analysed, together with documents published by legislative bodies. The key points for each topic are identified and described together with useful tips for precise safeguarding of MRI operators, in terms of exposure assessment, studies on biological effects, and health surveillance.

Keywords: Magnetic resonance, MRI, Electromagnetic fields, Occupational exposure, Exposure assessment, Regulations and guidelines, Health Surveillance

\section{Summary}

Abstract

1. Introduction.

2. Methods

3. Exposure assessment.

3.1. Exposure assessment of workers with implantable medical devices 7

3.2. Discussion

4. Biological effects due to exposure in MRI 10

4.1. Established mechanisms of interaction 
4.3. Discussion

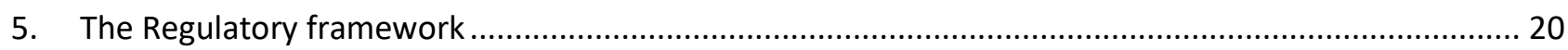

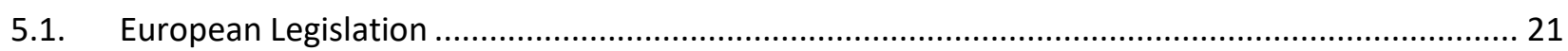

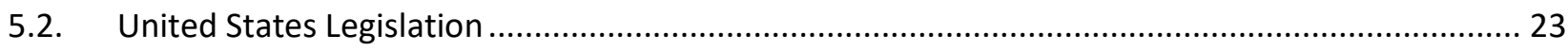

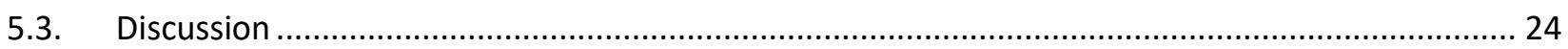

6. The health surveillance and the problem of the workers at particular risk: the European approach

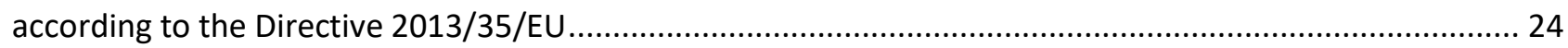

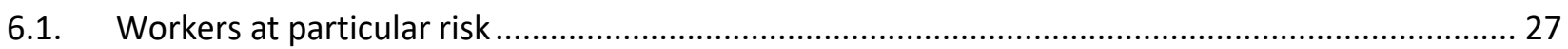

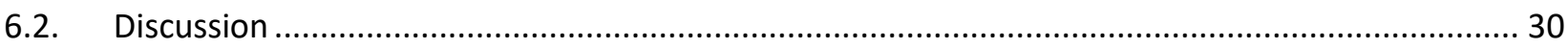

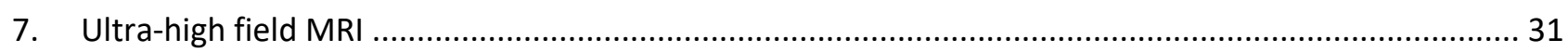

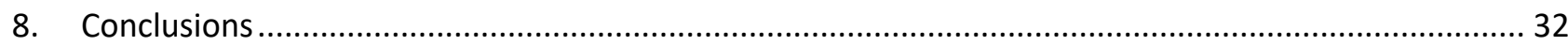

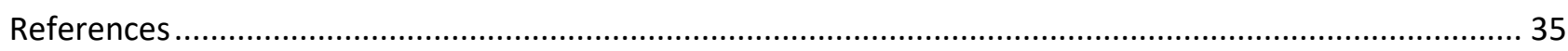

\section{Introduction}

Magnetic Resonance Imaging (MRI) is one of the most-used diagnostic imaging methods worldwide [1]. Since its first application on humans in the late 70ths, the evolution of MRI technology has been very rapid, and nowadays it is estimated that more than 60 million exams per year are performed worldwide [2].

There are $~ 50,000$ MRI scanners worldwide, and each MRI unit involves a minimum of five workers from different disciplines (radiographers, nurses, anesthesiologists, technicians, engineers, cleaners, etc.,) [3] These people may be exposed to various electromagnetic fields (EMF) during procedures, opening a debate about the occupational exposure to EMF in MRI environments [4]. Many self-reported transient symptoms have been associated with occupational exposure to MRI fields, especially from workers in MRI environments with a strong magnetic field (1.5 T, $3 \mathrm{~T}$, or higher) [5-8]. Several studies assessing the health 
risk for MRI workers have been published, showing different acute neurobehavioral effects and symptoms, but their long-term health significance is yet unknown. Chronic and long-term effects due to exposure to magnetic fields have been reported by only a few studies [9-12], but confirmation of these findings is still lacking. International organizations such as the International Commission on Non-Ionizing Radiation Protection (ICNIRP) $[13,14]$ and the Institute of Electrical and Electronic Engineers (IEEE) $[15,16]$ have established limits for occupational exposures. These international guidelines are based on the only adverse effects on humans that were fully verified by a stringent evaluation, i.e. short-term effects, such as stimulation of peripheral nerves and muscles, shocks and burns caused by touching conducting objects, and elevated tissue temperatures resulting from absorption of RF energy during exposure to EMF.

In 2013, the European Union issued a directive on occupational EMF exposure which has been implemented as a national standard in the member states [17]. The Directive is based on all known direct biophysical effects and indirect effects caused by EMF and it intends to ensure the health and safety of workers. From the point of view of radiological protection, the health risk related to MRI procedures is considered low compared to ionizing radiation. The strong static magnetic field (SMF), the switched gradient magnetic fields (GMFs), and the RF pulses, that are typical of MRI, are parts of the non-ionizing radiation in the EMF spectrum, and as such, their associated photons have insufficient energy to ionize the atoms of biological matter. The presence of static and low-frequency, and RF magnetic fields makes the assessment of exposure complex and the analysis of possible effects is a challenging task [4]. Moreover, a worker who moves through the spatial gradient of the static field (fringe field) is also exposed to a low-frequency time-varying magnetic field (motion-induced TvMF).

MRI technology has a continuous and very rapid development together with an increasing interest in its versatility for both clinical and research purposes. As a consequence, increased use of MRI scanners with strong magnetic fields is being pursued: a few institutions, also in Europe, have already installed human scanners that can reach $9.4 \mathrm{~T}$ [18]. Not to mention all the MRI scanners dedicated to animal studies, both for veterinary purposes and preclinical research, that generally are not subjected to the same regulations but often have a very strong magnetic field value. An ultra-wide bore magnet for studies on small living animals recently installed in US has a field strength of $21.1 \mathrm{~T}$, and it is the strongest MRI scanner in the world [19]. 
Safeguarding the health of workers in MR is a very complex task, still partially to be explored. It is important to approach this topic from the various points of view involved, from health risks and exposure assessment to regulations and health surveillance. The main purpose of this review is to analyse the state-of-art of literature about the several aspects of occupational exposure to EMF in MRI, identifying the key points for each topic. Finally, by this detailed analysis, useful tips for the precise safeguarding of MRI operators are deduced and presented. This work is an updating and integration of our previous review [20]: regulations, literature studies on biological effects, and health surveillance are addressed here in detail, along with a summary of the main approaches for exposure assessment. The specific problem of patient exposure and possible health risk related to MRI procedures is not considered here as it is widely treated elsewhere in the literature [2126].

\section{Methods}

We searched for relevant articles on occupational exposure to EMF in MRI indexed in Scopus and Web of Science (WoS) published from 2013 to 2021.

We carried out three widespread searches combining the following terms: (occupational exposure AND electromagnetic field AND magnetic resonance) OR (magnetic resonance AND worker AND electromagnetic field exposure) OR (MRI AND occupational exposure AND electromagnetic field) for the “exposure assessment" section; (electromagnetic field* OR static magnetic field* OR gradient field*) AND (MRI OR magnetic resonance) AND (biological effect* OR in vitro OR in vivo OR epidemiology) for the "biological effects" section; (magnetic resonance AND worker AND electromagnetic field exposure) AND (health OR prevention OR surveillance OR occupational medicine) for the "health surveillance" section.

No restrictions on source types were applied (i.e., article, book chapter, conference, report, letter, etc.). We also analysed the references list of the selected articles and we included additional older relevant contributes. In addition, specific research was conducted on several worldwide organizations' websites and databases to select the regulations in force about the topic of the review.

We screened the title and abstract of all the articles returned by the search. We reviewed the full text of the potentially relevant articles. We did not consider articles written in a non-English language. 


\section{Exposure assessment}

The main purpose of the exposure assessment is to verify the compliance with exposure limits set by the regulation in force, as well as to characterize possible exposure scenarios in the framework of epidemiological studies on MRI occupational exposure, that is recommended as a high priority to fill the gap in the knowledge on the related health effects [27]. The exposure assessment in MRI environments was described in our previous review [20], so in this present work, we summarize and update the main methods used for the assessment of exposure to each type of field.

For assessment of exposure to SMF, the physical quantity considered is the magnetic flux density (B, in $\mathrm{T}$ ). The unperturbed field in the MRI environment can be determined by using the isogauss line maps provided by the scanner manufacturers [28, 29], or by direct measurements using commercial survey meters [30-34]. Measurements of SMF exposure should be performed together with observational studies [35], which consist of recording, thorough daily diaries or questionnaires, the specific actions performed by the participants, in order to associate SMF exposure conditions to specific activities, and, eventually, to the reporting of transient symptoms [36].

Personal measurements can be conducted using time recording systems, often referred to as "dosimeters" which are generally worn by the workers in a pocket or fixed by an elastic strap around the breast, and measure the instantaneous value of B [37-42]. The new-generation personal dosimeter [43, 44] are able to evaluate also the exposure to motion-induced TvMF during daily clinical practice [33, 40, 41, 45-47].

Motion-induced TvMF can be assessed also using analytic models [31, 32, 34, 48, 49], as suggested by ICNIRP [50], or computational calculations [51-53], mainly based on Finite-Element Method (FEM) or the Boundary Element Method (BEM) [54, 55].

The measurement of GMF is commonly performed with specific commercial instruments [56-58]. Generally, the height of the probe above the floor has been chosen as representative of maximum exposure, for a simple "worst-case" approach to risk assessment.

Also, numerical solvers can be used to the assessment of GMF exposure close to the bore of a scanner, to obtain estimates of induced electric fields and current density [59, 60]. 
The measurement of RF fields in the proximity of MRI systems can be obtained by using commercial devices equipped with specific probes $[28,61]$. These devices provide also the RF harmonic content of a specific imaging sequence [62]. Direct measurement of Specific Absorption Rate (SAR) is still not possible with commercial instruments; a device for monitoring SAR has been proposed [63], but it is not sufficiently sensitive to monitor occupational RF exposure outside the magnet bore.

In order to estimate the SAR value in specific exposure conditions, it is possible to use numerical calculations and models of RF coil and the human body. The most used method is the Finite Difference Time Domain (FDTD) method [64]. Whole-body human models or models of specific parts of the body can be used in computational calculations [65]. Different adult and child, male and female models were developed within the Virtual Family Project on the basis of high-resolution MRI scans and include more than 80 tissues and organs each one [66].

At frequencies below $100 \mathrm{kHz}$, MRI staff experience magnetic fields with complex waveforms. This means that they are distorted by harmonic components, which are distributed over a large frequency band. This regards both motion-induced TvMF and GMF. Moreover, the safety limits imposed by the guidelines and regulations are frequency-dependent. In these conditions, parameters such as peak or root mean square values are inappropriate for exposure assessment. Therefore, both ICNIRP guidelines [50, 67] and EU Directive $[17,68]$ recommend the Weighted Peak Method (WPM) for assessing compliance with exposure limits in case of complex waveforms. WPM can be implemented either in time-domain (hardware or software implementation) $[56,57,69]$ or in frequency-domain $[48,70]$. The result of this approach is the WP index, whose value must be below 1 to ensure compliance.

Some commercial instruments permit the implementation of the weighted-peak approach [56, 57], according to the ICNIRP 2003 [71] statement on complex waveforms and the ICNIRP 1998 [13] reference levels.

\subsection{Exposure assessment of workers with implantable medical devices}

Workers with implantable medical devices, especially with an active implantable medical device (AIMD) such as cardiac pacemakers (PM), cardiac defibrillators (ICD), cochlear implants, brainstem implants, neurostimulators, retinal encoders, implanted drug infusion pump, are considered as a particularly sensitive 
risk group (see also Section 6.1). Indications for the risk assessment of workers with AIMD can be found in the "Non-binding guides" of the European Commission (EC) $[68,72]$, which recall the instructions described in the EN50527 technical standards family [73-75]. According to the standards and the new European Medical Device Regulation [76] each AIMD shall comply with the electromagnetic immunity requirements, before entering the market. Thus, if a worker who wears an AIMD is exposed to EMF levels below the ICNIRP reference levels for the General Public [13], the risk is considered acceptable. A safety value of 0.5 $\mathrm{mT}$ is specified to limit interference with the function of active implanted medical devices and a maximum value of $3 \mathrm{mT}$ is indicated to limit the projectile risk in the fringe field from strong sources (> $100 \mathrm{mT})$.

Volume 1 of the "Non-binding guides" identifies the working conditions that require a specific exposure assessment for workers wearing active implants, due to the presence of strong fields close to the device [68]. MRI is one of these conditions and hence requires specific risk assessment in accordance with the specifications provided in Annex A of the EN50527 standard [73].

There are some studies in the literature on the EMF exposure assessment of workers with active implants, mainly to avoid interference problems with, or effects on the functioning of, medical devices [77-79].

In one of these, Mattei and colleagues [78] evaluated experimentally the effect of the movement near the MRI scanner bore for workers with a PM or an ICD, both marked as MRI-conditional [80]. They used a saline-filled human-shaped phantom [79], equipped with different kinds of medical implants, an accelerometer and a magnetic field probe. Then, the authors reproduced both translational and rotational motions close to the scanner measuring acceleration and magnetic field variation generated by the movement of the phantom. The results of the study demonstrated that function of both implants tested was influenced by electromagnetic interference from motion in and around an MRI scanner, leading to inappropriate changes in therapy. Other kinds of AIMDs would deserve a specific exposure assessment, such as the cochlear implants which generally use inductive coupling as part of the normal function and could be consequently susceptible to interference in the presence of specific fields. The safety of these kinds of implant in patients undergoing MRI examination is widely discussed [81, 82], but to date, there is a lack of specific studies about exposure assessment of workers with cochlear implants.

Passive implants, (i.e. knee and hip prostheses, copper intrauterine device (IUD), etc) contain no electric parts and they are normally constituted by non-ferromagnetic materials like titanium or stainless steel. 
Several studies in literature dealt with the exposure assessment of MRI patients with passive implants [8385], but there are no specific studies about exposure assessment of MRI workers with this kind of implant. Since there are in the literature some recent studies about transient health effects in MRI workers with such kinds of implants, for example, IUD [86, 87], this specific issue would deserve more attention (see also Section 6.1).

\subsection{Discussion}

At the moment, there are no standardized procedures to assess the MRI occupational exposure, although ICNIRP has proposed a method to verify the compliance of the exposure parameters with the safety limits set for the exposure due to movement in a non-homogeneous SMF and to the GMF [50].

Numerical approaches to the assessment of the MRI exposure are, currently, an invaluable tool mainly to evaluate the interaction of EMF with implanted medical devices, such as cardiac pacemakers and defibrillators, insulin pumps, metallic prostheses, cochlear implants, etc [83]. The recent and rapid development in new numerical algorithms combined with relatively low-cost powerful computational resources (e.g., graphic processing units), have favoured the spreading of these algorithms, also thanks to the availability of increasingly sophisticated numerical anatomical human models, with an accurate characterization of dielectric properties of different tissues. However, numerical simulations for the exposure assessment can be affected by worker body model characteristics including posture, dimensions, shape, and grounding conditions [88]. The working environment models including objects influencing field distribution can significantly under- or overestimate the exposure effects in the body.

Exposure assessment using a personal measurement system, such as dosimeters, has some technical and ethical limitations mainly due to covering the real working shift which includes work with patients. The large variability among patient examinations causes that the parameters measured in any work scenario have only limited representation of the real situation [33]. Due to this wide variability, the direct comparison of results from different studies completed by adopting different assessment strategies is difficult, or often not possible. Defining a baseline of standardization for the methodologies for collecting exposure data at a personal or at environmental level is mandatory, together with a detailed analysis of the specific tasks and procedures adopted, using, for example, information from observational studies, to obtain a better 
characterization of the exposure conditions and to identify the factors determining such exposure variability. Finally, in personal monitoring of exposures, the positioning of the sensing probe on the body of the worker is also very important: it has been shown that the exposure measured at the upper body is likely to be higher because more rotational movements are made, and staff leans forward during certain tasks, putting their torso closer to the magnet bore [69].

\section{Biological effects due to exposure in MRI}

MRI is generally considered safer than nuclear imaging technology or X-ray procedures for humans because it does not require the use of ionizing radiation, for which the harmful effects on human health are very well known [89]. MRI devices employ EMF of three different types to produce images [1], such as a strong SMF, the gradient magnetic fields, and the RF fields whose associated energy is insufficient to ionize the atoms of biological matter.

The static magnetic field is always turned on in the MRI suite irrespective of whether the scanner is working or not, thus it represents the main source of MRI-related exposure due to the stray field surrounding the scanner. The magnetic induction level in the patient area ranges from $1 \mathrm{~T}$ to several $\mathrm{T}$ for different scanners. Due to the active shielding of the SMF, the field decreases quickly with a distance from the scanner, producing a large gradient with the field becoming significant within $0.5 \mathrm{~m}$ from the bore opening. Movements of the personnel in the spatial gradient of the SMF cause exposure to induced TvMFs [4, 90]. Exposure to GMFs and RF fields takes place only in special cases when the MRI workers stay close to the scanner during imaging acquisition such as during dynamic examinations, in emergencies, while attending paediatric patients, or for interventional medical procedures [67, 91-93].

The GMFs, originating from three separate coils within the scanner, are switched on and off to select the region of diagnostic interest and to spatially encode the MRI signals.

In this paragraph, we briefly describe the well-established physical mechanisms of interaction between the MRI-related EMF and living tissues [94], that are responsible for acute effects, i.e. transient effects occurring above threshold exposure levels which provide the basis for the definition of the exposure limits. Moreover, we report on the possible health risk of exposure to EMFs used in MRI below the thresholds of acute effects, which rely on the results from the three main lines of evidence that are epidemiological, in vivo, and in vitro 
studies. In particular, we provide an overview of the effects arising from multiple exposures taking place when staff personnel operates inside the MRI suite [91], and from a single exposure to SMFs and GMFs. There are no data available on RF signals currently in use for MRI. It is worth mentioning that although the focus is on occupational exposure, studies carried out on patients and volunteers have been included since they report on the effects of multiple exposures.

The main conclusions of the most recent reviews of the epidemiological, in vivo, and in vitro evidence, released by the Scientific Committee on Newly Identified Health Risks (SCENIHR) in the last Opinion [27], and updated with the most recent studies will be presented.

\subsection{Established mechanisms of interaction}

\section{Static fields}

The three main physical mechanisms of interaction between SMFs and biological tissues are magnetic induction, magneto-mechanical, and chemical interactions $[1,24,95]$. The magnetic induction effect is considered responsible for the sensations of nausea and vertigo sometimes reported at high field strengths [4]. In MRI workplaces, movement through the gradient of the static field (fringe field) acts as a timevarying magnetic field (motion-induced TvMF) [50, 67] inducing a voltage in electrically conductive materials, like biological tissues, according to Faraday's law [21]. Hence, rapid movements of the body induce a great electric field in the tissue and this can cause a number of physiological symptoms (headache, nausea, vertigo, phosphenes, numbness and tingling, loss of proprioception and balance) [96].

Magneto-mechanical interactions refer to the mechanical torque and force that an SMF can exert on ferromagnetic objects, such as metal of high-magnetic susceptibility. This fact represents an extremely important issue in the case of medical devices implanted in the human body (clips, vascular or orthopaedic implants) (see also Section 3.1) [1].

Chemical interactions refer to changes in chemical reactions due to altered spin chemistry. This direct interaction mechanism is not considered to have a significant health effect for magnetic flux density below 7 $\mathrm{T}$, whereas above $7 \mathrm{~T}$ there is too little research to draw firm conclusions [95].

\section{Gradient magnetic fields}


Generally, GMFs are about two orders of magnitude lower than the SMF and can be neglected compared to the strong $\mathrm{B}_{0}$ field as far as interactions with biological tissues and organisms are concerned. However, the gradients are switched on and off rapidly (at frequencies in the $\mathrm{Hz}$ to $\mathrm{kHz}$ range [23]) and this time variation induces electric currents in the exposed body [21]. If the induced electric currents exceed the nerve depolarization threshold, peripheral nerve stimulation (PNS) can occur [92].

The induction of electric currents in the retina can cause the phenomenon of magnetophosphenes, which are not considered to be hazardous to humans but can impair the working ability.

\section{Radiofrequency Fields}

As in the case of GMF fields, exposure to RF fields exclusively occurs in proximity to the bore entrance and only during scanning.

The primary bio-effects associated with the RF radiation used for MRI procedures are thermal effects due to tissue heating derived by the direct absorption of electromagnetic energy. This heating can have possible physiological effects, including changes in cardiac output, and alteration in certain organs particularly sensitive to heating due to lack of perfusion, such as the eyes and testes [97]. The amount of the energy absorbed by the body is measured in terms of SAR that is the dosimetric parameter used to quantify the absorption at RF [4].

In the presence of conducting materials close to the exposed body, such as the leads of equipment for monitoring physiological parameters (heart rate, blood pressure, oxygen saturation, and temperature), more caution is required to avoid excessive local heating [98]. This kind of risk can be more serious in the case of internal biomedical implants (aneurism clips, stents, etc.), especially for implants that have elongated configurations and/or are electronically activated (neurostimulation systems and cardiac pacemakers) [22, 99]. For more details on this topic, the reader can refer to the specific literature $[83,100,101]$. See also Section 3.1 and Section 6.1.

\subsection{Experimental and epidemiological studies on the effects of EMF exposure in MRI}

\section{Effects of multiple exposures}


While epidemiological studies on multiple exposures in MRI were not retrieved, most of the studies which address the occupational health risk due to the multiple exposures in MRI environment, have been carried out on volunteers exposed to MRI sequences in clinical scanners.

In Schlamann et al., 12 healthy volunteers were exposed for 63 min to standard sequences in a $1.5 \mathrm{~T}$ MRI scanner to investigate possible cognitive effects using transcranial magnetic stimulation performed immediately after exposure and 15 min later. This procedure was repeated 4 weeks later using a $7 \mathrm{~T}$ scanner. At both field intensities, the cortical silent period was significantly prolonged to become normal after 15 min. The transient effect elicited at both 1.5 and $7 \mathrm{~T}$ can be ascribed to RF pulses and/or the gradient fields rather than to SMF [102].

The effects on male reproductive hormones were investigated in 24 healthy, male volunteers exposed to 1.5 $\mathrm{T}$ MRI. No differences were observed in the serum blood level of a series of key hormones neither immediately after, nor after 11 days of MRI and sham exposure [103].

Thulborn et al did not find significant changes in the vital signs or cognitive performance in either healthy subjects (22) or subjects with medical diagnoses (24) subjected to brain MRI examination at $9.4 \mathrm{~T}$ and examined before and after the scan [104].

In van Nierop et al., neurocognitive effects resulting from exposure to SMF alone and combined with lowfrequency TVMF in a 7 T MRI scanner were investigated. 36 healthy subjects were exposed in four sessions of 2 exposure conditions and 2 sham conditions in front of the scanner bore (1.0 T SMF with or without 2.4 T/s TVMF, induced by standardized head movements before each of the five neurocognitive tasks). Neurocognitive effects were only observed in the case of simultaneously exposed to SMF and TVMF from a 7 T MRI scanner [105].

Cognitive, vestibular, and physiological functions were investigated in a recent study by Grant and coworkers. A total of 29 subjects were exposed to 10.5 T MRI and tested for multiple physiologic, cognitive, and vestibular markers before, during and after exposure (1-24 h). Moreover, for 26 subject's exposure and testing were repeated within 2-4 weeks. Several comparisons were made between short- and long-term time points with respect to the parameters. The results indicated that cognitive performance was not compromised at the isocenter; subjects experienced increased eye movement at the isocenter, and subjects experienced 
small changes in vital signs but no field-induced increase in blood pressure. While small but significant differences were found in some comparisons, none were identified as compromising subject safety [106].

In several studies, genotoxic effects in peripheral blood samples from healthy volunteers or patients exposed to clinical MRI scanners matched with appropriate controls were investigated. Mainly cardiac MRI (CMR) was employed, due to the high gradient-fields applied in this specific examination, as well as the large portion of the body exposed to RF and SMF during the procedure. Widely recognized cellular endpoints that account for chromosomal damage such as micronuclei (MN) formation and chromosomal aberration (CA), DNA damage and repair such as induction of double-strand breaks (DSBs) by means of $\gamma$-H2AX foci formation and comet assay, were investigated.

Sannino et al, in a pilot study, did not find spontaneous and mitomycin-C-induced chromosomal fragility in human peripheral blood lymphocytes from 12 MRI workers matched with 12 workers with no MRI exposure history, as the reference group. The results also highlighted a high worker-to-worker variability in both exposure assessment and biomonitoring, as well as the critical issues and practicalities to be faced within this type of investigation [48].

Simi and co-workers reported on an increased $\mathrm{MN}$ formation in lymphocyte cultures from healthy volunteers established immediately after four consecutive sequences in a $1.5 \mathrm{~T}$ MRI scanner and a CMR examination. The MN frequency was approximately doubled immediately after the scans but returned to control level at $48 \mathrm{~h}[107]$.

Fiechter and co-workers studied genotoxic effects in twenty prospectively enrolled patients who underwent 1.5 $\mathrm{T}$ cardiac MRI examination. They found a statistically significant increase in $\gamma$-H2AX positive foci in peripheral mononuclear cells and in T lymphocytes that were indicative of sites of DNA DSBs repair [108]. Lancellotti and co-workers examined blood samples from twenty enrolled healthy men subjected to $1.5 \mathrm{~T}$ CMR. They found blood cell alterations or activations figuring inflammatory response, as well as DNA damage in T lymphocytes from day 2 after exposure until the first month. These effects disappeared at 1-year follow-up [109].

Brand and co-workers did not found DNA DSBs in blood samples from forty five patients that underwent CMR with different protocols by investigating $\gamma-\mathrm{H} 2 \mathrm{AX}$ foci formation [110]. 
Fatahi and co-workers examined the extent of genetic damage in eleven healthy, non-smoking, male subjects, who had never undergone radio-/chemo-therapy, scintigraphy, and had not undergone X-ray examination one year before blood sampling. They were repeatedly subjected to $7 \mathrm{~T}$ and $3 \mathrm{~T}$ MRI during their working life (from 2.5 to 8 years), and $\mathrm{MN}$ and $\gamma \mathrm{H} 2 \mathrm{AX}$ foci formation were analyzed. No difference in the cellular endpoints was detected in samples from exposed subjects with respect to the unexposed ones. Isolated cells were further exposed in vitro to $7 \mathrm{~T}$ MRI either alone or in the presence of the DNA damaging drug, etoposide, and the kinetics of DNA damage repair was examined to determine if there was any additional combined effect. Also in this case, $7 \mathrm{~T}$ MRI had no significant impact on MN frequencies and $\gamma \mathrm{H} 2 \mathrm{AX}$ foci at 1,20 and $72 \mathrm{~h}$ after exposure [111].

Reddig and co-workers examined the impact of different magnetic field strengths $(1,1.5,3$, and $7 \mathrm{~T})$ and the effect of contrast agent on DNA DSBs formation in forty-three patients undergoing MRI. No evidence of DSB induction after MRI examination, independent of the applied field strength and administration of gadolinium-based contrast was found [112].

Fasshauer and co-workers, by evaluating $\gamma \mathrm{H} 2 \mathrm{AX}$ foci formation did not find impairment in DNA integrity in the mononuclear cells from thirty-six volunteers undergoing $1 \mathrm{~h} 3 \mathrm{~T}$ CMR examination and analyzed $1 \mathrm{~h}$ after or after $2 \mathrm{~h}$ of leisure time without strong physical activity [113].

Suntharalingam and co-workers, investigated $\gamma \mathrm{H} 2 \mathrm{AX}$ foci formation in human peripheral blood lymphocytes of two cohorts of twenty healthy volunteers each subjected to abdominal-pelvic MRI performed at $1.5 \mathrm{~T}$ and 3.0 T. No significant alteration in the endpoint analyzed was found 5 and $30 \mathrm{~min}$ after scan [114].

Contrast enhanced abdominopelvic 3T MRI was also employed by Jalali and co-workers to investigate chromosomal damage in the MN test and the expression of some genes involved in DNA repair and apoptosis pathways in lymphocytes from 20 enrolled healthy subjects. No effect was detected in the endpoints under investigation at 2 and $24 \mathrm{~h}$ after MRI examination [115].

Critchley and co-workers investigated the acute effect of $1.5 \mathrm{~T}$ CMR lasting $40 \mathrm{~min}$ in a large cohort of sixty-four patients immediately before and after a standard clinical scan. The CMR was not associated with a significant change in $\gamma \mathrm{H} 2 \mathrm{AX}$ foci formation in leucocyte samples, although there was significant interpatients variability [116]. 
The effects on testis histology and seminiferous tubules morphometry were investigated by Rostamzadeh and co-workers in a group of 20 mice exposed for 36 min once a week for three weeks to $1.5 \mathrm{~T}$ MRI under different pulse sequences A group of 20 mice located in the center of MRI bore while it was turned off was considered as control. Different assays were performed at $1 \mathrm{~h}$ and 35 days after last exposure, and deleterious effects on male reproductive system, fertility parameters, and the quantity of germ cells in the seminiferous tubules were recorded that reverted to normal after a new period of spermatogenesis [117].

Genotoxicity in blood cells from healthy donors was also the most investigated outcome of in vitro studies.

Lee and co-workers investigated the frequency of CA and MN and the extent of DNA migration in the comet assay in human peripheral blood lymphocytes from one donor exposed from 22 to 89 min to a $3 \mathrm{~T}$ MRI scanner. An increase in MN formation, CA and in the extent of DNA migration was detected, although it resulted time-dependent in the first two assays [118]. Similar experimental conditions were applied by Szerencsi and co-workers, who exposed peripheral blood samples from three healthy donors to 3T MRI for $0,22,45,67$, and 89 min during the scanning procedure. No effects were detected in DNA migration and CA [119].

Reddig and co-workers did not find DNA DSBs in human peripheral blood mononuclear cells isolated from sixteen healthy donors and exposed to 7T SMF alone or combined with varying gradient magnetic fields and RF pulses. In follow up studies, the same research group investigated the effects of ultra-high-field 7T MRI in mononuclear cells from twelve healthy donors [120]. They did not find effects on DSBs in un-stimulated cells at 1, 20 and $72 \mathrm{~h}$ post exposure and on MN formation in mitogen-stimulated lymphocytes cultured for $72 \mathrm{~h}$ [111]. They also examined the possible amplification of the genotoxic and cytotoxic effects of different gadolinium-based contrast agents in combination with ultra-high-field 7T MRI exposure in isolated human peripheral blood lymphocytes. They failed to detected any alteration in $\gamma \mathrm{H} 2 \mathrm{AX}$ foci formation in lymphocytes from four donors analyzed $15 \mathrm{~min}$ after the scan [121].

Critchley and co-workers, investigated the acute effect of $1.5 \mathrm{~T}$ CMR lasting $40 \mathrm{~min}$ on leucocyte DNA integrity from seventeen healthy donors, and no alteration in $\gamma \mathrm{H} 2 \mathrm{AX}$ foci formation was detected [116].

Comprehensive reviews on the possible genetic damage due to MRI exposure have been published which accurately comment on the published in vitro and in vivo studies above cited with the main focus on biological outcome [122-124] and on MRI exposure related aspects [125, 126]. In these review papers, the 
strength and methodological limitations of the studies are highlighted together with the research need to have good quality studies, including the epidemiological studies addressing the potential health risk for MRI workers.

\section{Effects of SMFs exposure}

There are only a few epidemiological studies available, and the majority of these have focused on cancer risks. Results did not indicate any increased risk for cancer development, but they are generally based on small number of cases and not accurate exposure assessment. Moreover they lack of the control of confounding factors in occupational setting, that often are due to other harmful exposure sources including some known carcinogens like ionizing radiations, chemicals can be exposed to during their work shift not necessarily only in MRI procedure [127].

From the observational studies published before 2015 and discussed in the SCENIHR opinion [27] it came up that the movement in strong SMF (field strengths above $2 \mathrm{~T}$ ) may cause subjective symptoms like vertigo and nausea. Of note is the systematic review and meta-analysis carried out by Heinrich and co-workers in which studies from 1992 to 2007 were analysed to evaluate whether cognitive processes, sensory perception, and vital signs might be influenced by SMF in MRI environment. Only effects on the visual system were homogeneous, showing a statistically significant impairment following exposure. Vital signs were not affected, and effects on sensory perceptions included an increase of dizziness and vertigo, primarily caused by motion-induced TvMF [128]. Similar results on symptoms like dizziness, vertigo and nausea have also been reported in more recent papers. De Vocht and co-workers found out that prevalent symptoms were associated with longer duration of working in MRI departments, but not with measured field strength of exposure. Other factors related to organization and stress seem to contribute to increased reporting of these symptoms [129]. Schaap and co-workers reported increased vertigo with increasing strength of the static field in people working around scanners [130]. Walker and co-workers, examined the symptoms experienced by 408 MRI technologists from multiple countries, acquired by means of questionnaire administered via Facebook. From the descriptive statistics, it came up that $78 \%$ of participants experienced undesirable symptoms related to SMF exposure. Dizziness, vertigo, visual disturbances, nystagmus, and metallic taste were the most frequently reported symptoms. Dizziness was the most frequent symptom, and its severity was greater among technologists who worked with very high and ultra-high field strength compared to those who 
worked with low-field and high-field strengths. Participants in the study also reported symptoms never reported previously, such as pain, weakness, numbness, and dropping things [8].

A huge amount of research papers, review papers, and book chapters has been published to address the possible biological interaction of magnetic fields with in vivo and in vitro systems after moderate (1mT 1T), strong (1 - 5T) and ultra-strong (>5 T) and different exposure duration from minutes to days, mainly based on the analyzed endpoints. In vivo studies mainly addressed embryonic development, nervous system effects, behavior and various physiological parameters and metabolism in laboratory animals. In a number of studies, effects occurred with SMF exposures in animals, at B field levels from $\mathrm{mT}$ to $\mathrm{T}$, but they are limited to single studies in the specific area, and need replications prior to draw any conclusion. Recent papers contradict the effects on nervous system reported in some studies over the years. The studies that report pain reduction are consistent and in line with previous studies by the same group. The studies on behaviour, carried out also at very high field strength, did not produce consistent effects, like the ones focusing on lipid metabolism [27].

More recent studies on high field strength do not demonstrate severe long-term effects on mice. Mice exposed to SMF in the range 3.5-23 $\mathrm{T}$ for $2 \mathrm{~h}$ did not show any effects in terms of food/water consumption, blood glucose levels, blood routine, blood biochemistry, as well as organ weight and HE stains after daily examination until 3 weeks [131]. Moreover, 28 days exposure of mice to SMF in the range 2-12 T did not exert effects on body weight, blood indices, organ coefficients, and histomorphology of major organs [132]. The in vitro studies addressed several cellular endpoints including genotoxicity, oxidative stress, gene and protein expression, apoptosis, cell viability, growth and differentiation and membrane effects. The results of the most recent studies confirm the ones of previous investigations summarized in several review papers $[133,134]$. Alteration in the expression of specific genes in human and other mammalian cells have been reported, with the effect depending on exposure duration and field gradients. Genotoxicity have been reported, although it seems that these effects can be repaired and are not permanent. There are also evidences that SMF can modify the effects of other external factors such as chemicals and ionizing radiation. All the effects strictly depend on cell type and exposure duration and characteristics [27].

Conflicting results are also reported in more recent investigations. Romeo and co-workers failed to find effects on cell viability, reactive oxygen species levels and DNA integrity in human foetal lung fibroblasts 
exposed to $370 \mathrm{mT}$ magnetic induction level, under different exposure regimens. In this study, an experimental apparatus designed and realized for operating with the SMF generated by permanent magnets, and confined in a magnetic circuit, was used to allow cell cultures exposure in absence of confounding factors like heating or electric field components [135].

Permanent magnet was also used by Tian and co-workers to examine the effects on cell growth of 12 different human cancer and non-cancer cell lines exposed to 0.2-1 T SMFs with different magnet directions. Different magnetic field directions gave divergent effects with upward magnetic field mostly capable to reduce cell growth of cancer cell lines [136]. Zhang and co-workers evidenced no effect of $1 \mathrm{~T}$ SMF on cell cycle or cell death of 15 different mostly human cell lines. However, at higher cell density, SMF reduced cell numbers in six out of seven solid human cancer cell lines indicating the cell type and density-dependent effect [137].

\section{Effects of switched gradient fields}

Only a few studies have been conducted in the intermediate frequency range that is related to GMFs. In [27], the absence of epidemiological studies was highlighted, while the few relevant in vivo and in vitro studies did not allow health risk assessment. These conclusions were confirmed by a recent review paper by Bodewein et al [138]. The authors performed a systematic review of the studies of electric fields, magnetic fields and EMF in the frequency range $300 \mathrm{~Hz}$ to $1 \mathrm{MHz}$ to identify any potential risks. They identified 3 human studies investigating the effect of MF exposure $(0.1 \mu \mathrm{T}$ to $2.8 \mathrm{mT})$ on human visual and cognitive functions. Two of them $[139,140]$ addressed specifically the effects of $490 \mathrm{~Hz}$ MF generated by MRI on the visual system, and found no statistically significant differences between exposure and control conditions. Moreover, in 27 in vivo studies, mainly effects on reproduction and development, brain, cardiovascular system and hematological parameters and behaviour, were analyzed. In the identified 25 in vitro studies, effects on cell proliferation and cell viability, genotoxicity and gene expression, were considered. The general conclusion was that there are inadequate evidences for adverse effects due to a large heterogeneity in the analyzed species, applied frequencies, field strengths, exposure durations and examined endpoints [138].

\subsection{Discussion}


Apart from the established acute effects related to EMF exposure in MRI, evidence regarding the possible effects of exposure below the thresholds of acute effects are not sufficient for a proper health risk assessment for the MRI workers [94]. Only a few epidemiological studies have been carried out [27]. They all address the effects of SMF exposure in terms of cancer risk but they lack accurate exposure assessment and control of confounding factors in occupational settings. From observational studies, prevalent symptoms like dizziness, vertigo, and nausea are the most reported effects that are associated with longer duration of working in MRI departments [128]. As far as the experimental studies are concerned, they do not provide consistent evidence due to great variability in terms of biological model, exposure characteristics, and biological outcome. In vivo and in vitro effects on DNA integrity are the most investigated and clearly of interest to follow up because excess genetic damage in somatic cells can lead to carcinogenesis, and in germ cells can be transmitted to the next generations. However, in the sporadic cases in which an effect was found under multiple exposure conditions, it is not clear to which component of the MRI exposure the effect can be ascribed [122]. In a very recent paper, Wilen and co-workers commented on the exposure conditions of in vivo and in vitro studies addressing the genotoxic effects of MRI exposure in human blood cells that vary greatly and often are not sufficiently described. They also highlighted the importance of having a detailed description of the MRI exposure to make the experiments repeatable in different laboratories [126].

\section{The Regulatory framework}

To optimize prevention and protection strategy, it would be appropriate to focus most significant risk scenarios and list of rules and procedures to be adopted in presence of MRI equipment, based on international guidelines and national safety regulations content.

The potential risk scenarios related to the use of MRI equipment therefore concern different categories of people directly or indirectly involved in the diagnostic activity:

- patients;

- the different types of workers who serve at the MRI site (health workers, cleaners, maintenance workers, helium refill operators, researchers); 
- visitors, or people who may find themselves accessing the MRI site and within the risk areas such as, for example, any accompanying patients, but also the staff of the health facility who is accessing the MRI site for particular reasons (internal audits, inspections, quality checks, training initiatives, etc.);

- critical population groups, that is, all those who inside and outside the healthcare facility (neighbouring areas) may be, even unconsciously, affected by the presence of the MRI tomograph, with particular reference to the presence of superconducting magnets.

Beyond ICNIRP and IEEE guidelines, electromagnetic field technical standards have been developed by the International Electrotechnical Commission (IEC) [141, 142], the European Committee for Electrotechnical Standardization (CENELEC) [143] and by other standardization authorities. Generally, these standards can be related to the EMF emission from equipment and provide instructions for the manufacturer to prove the safety of their products (emission standard) or provide standardized procedures for assessing the exposures in specific industrial environment (exposure assessment standards).

Generally the MRI industry-specific standard IEC 60601-2-33 has been used by the MRI community for both patient and occupational exposure limitation [142].

\subsection{European Legislation}

The current European regulations allow to frame, manage and, where possible, prevent the specific risks, as well as dictate the rules of conduct both for the operators directly concerned, and for all the other subjects who may be indirectly involved, knowingly or even unconsciously. They are summarized in Fig. 1, and represent the state of the art at the date of submission of this publication.

In 1999 the Council of the European Union published a Recommendation on limiting exposure of the general public to EMF (1999/519/EC) [144] for the protection from the proven adverse health effects of the exposure to EMF. The Recommendation is still in force, but it does not address the protection of workers.

European Union set the general arrangements for ensuring the health and safety of workers in the Framework Directive (1989/391/EC) [145]. In addition, EU more recently published the EMF Directive (2013/35/EU) [17] for the specific situation of work with EMF, with additional detail on how to achieve the objectives of the Framework Directive for this work condition. The EMF Directive is related to well established direct and 
indirect effects (acute or short-term effects) caused by exposure to EMF, according to the previous guidelines published by the ICNIRP (Fig. 1).

EMF Directive sets exposure limit values (ELVs) for sensory and health effects. Since the ELVs are generally specified in terms of internal body quantities, Article 3 of the Directive introduces action levels (ALs) which are external field quantities that can be easily measured or calculated. ELVs and the relative ALs are set according to the ICNIRP guidelines.

EMF Directive states that it is a responsibility of the employer to ensure that the risk arising from the specific case of EMF in the workplace is eliminated or reduced to a minimum. Moreover, the employer shall ensure the proper training of workers who are likely to be exposed to risks form EMF, concerning in particular the associated possible risks, the values and concepts of the ELVs and ALs, and the preventive measures taken. For the specific working environment of MRI, Article 10 of the EMF Directive grants a conditional derogation, meaning that "exposures relating to installation, testing, use, development, maintenance of, or research related to MRI for patients in the healthcare sector may exceed the ELVs". For the derogation to be valid, the risk assessment shall have shown that the ELVs are exceeded and the employer shall demonstrate that "workers are still protected against adverse health effects and safety risks, including ensuring that instructions for safe use provided by the manufacturer are followed".

The European Commission published two non-binding guides $[68,72]$ to assist employers to comply with the EMF Directive: the Volume 1 of these guidelines [68], provides practical guidance to employers of MRI facilities, to achieve compliance with the conditions of the specific Directive derogation. Moreover, the guides include the relevant ICNIRP publication of 2014 [50], containing guidelines for the protection of workers moving in the SMF.

The indications included in the two non-binding guides do not necessarily ensure the compliance with the legal requirements of each EU Member State, which always have precedence and can be more restrictive.

\section{Fig. 1 here}

In many countries of the European Union, national regulations relative to MRI clinical use (Table 1) were already aligned with the international technical standards before publication of Directive 35/2013. For these 
countries, the transposition of the directive did not lead to significant changes within MRI management. On the contrary, for countries that in 2013 were still tied to old ICNIRP guidelines (published before 2009), the transposition of the directive has definitely allowed an alignment to the harmonized technical standard suggested by the Directive. Italy represents an exception because in last thirty years it has dedicated some different specific decrees, sometimes defining safety rules stricter than International Standard of Good Practice suggested by ICNIRP or IEC.

In March 2020 ICNIRP published the new guidelines for human exposures in the RF region (100 kHz to 300 $\mathrm{GHz}$ ) [146] to replace previous guidelines [13], but for MRI nothing changes. New ICNIRP guidelines indicate the exclusion of pregnant staff from the MRI examination room during acquisition based on the possible RF exposure, but these workers are already excluded to avoid the risk to fetal hearing from the acoustic noise.

The Italian situation represents an exception in the European contest, due to the adoption of specific national standards, more detailed and stricter than the consolidated international guidelines, useful to define a prevention and protection system with the force of law [147, 148].

\section{Table 1 here}

\subsection{United States Legislation}

With regard to the management of safety in MRI in the United States, the debate has been going on for 20

years, finding particular impetus in correspondence with the accidents that have occurred at health facilities within the MRI departments.

The Food and Drug Administration (FDA), in its specific and indisputable role of "regulatory body", has formalized the definition of medical device for MRI tomographs, but to date it does not impose univocal rules with legal force for the management of safety related to them. As a consequence, during installation, the adoption of safety devices is left to the sole responsibility of those who install the tomograph. The guidelines and indications of the American College of Radiology (ACR) can be adopted but not required by law [149]. 
After the last fatal accidents occurred to paediatric patients between 2005 and 2012 [150], the process of issuing a regulatory code dedicated to MRI technology is currently in the evolution phase, but in any case, the ACR guidelines have become more punctual and composite over time, also on issues related more closely to non-clinical safety aspects, and their authoritativeness have propitiated a diffusion that presents an acceptable level of systematic demands.

\subsection{Discussion}

According to the current regulatory framework, the employer shall assess all risks and determination of the exposure and, especially in MRI environments, measure and/or calculate the levels of electromagnetic fields to which workers are exposed. Moreover, the employer shall take the necessary actions to eliminate or reduce to a minimum the risk arising from EMF at the workplace. Regarding the European scenario, the most of countries of the European Union has drafted the own national regulations relative to MRI clinical use in line with the international technical standards to then transposed the EU Directive 35/2013 [17]. The US regulatory framework is almost entirely based on technical standards [149].

However, there is a lack of specific regulations worldwide for MRI environments, both for clinical and research uses [1].

6. The health surveillance and the problem of the workers at particular risk: the European approach according to the Directive 2013/35/EU

In our previous review a relevant aspect, i.e. the Health Surveillance (HS) [151] of occupationally exposed MRI operators, was not addressed.

In the European Union the HS of these workers is a legal obligation according to the Directive 2013/35/EU. We will present here the approach to this issue currently practiced according to the legislations in force in Europe. According to the article 8 of this Directive: "with the objective of the prevention and the early diagnosis of any adverse health effects due to exposure to electromagnetic fields, appropriate health surveillance shall be carried out". Explicitly the Directive "is intended to address all known direct biophysical effects and indirect effects caused by electromagnetic fields", and "does not address suggested 
long-term effects of exposure to electromagnetic fields, since there is currently no well-established scientific evidence of a causal relationship". According to the Directive, the established effects to be prevented in MRI operators are the direct biophysical and the indirect effects. Examples of the former are the stimulation of muscles, nerves or sensory organs (including temporary annoyance or effects on cognition) and limb currents and, in case, the thermal effect. Among the indirect effects, projectile effect, explosions and fires risks are not preventable with HS programs, whereas interference and contact currents, and possibly also ferromagnetic effects for passive implants, should be addressed in HS programs by adequate counselling of the workers.

Regarding direct effects, it should be considered that a strict respect of the exposure limit values (ELVs) is sufficient to prevent the adverse effects, but it has to be noted that an exceeding of the ELV does not necessarily means the occurrence of adverse effects in MRI operators. In fact, in principle, ELVs are derived from scientific experimental evidence and epidemiological data (if available), applying appropriate reduction factors [152]. In the case of EMF, excluding the specific case of workers at particular risk, exposures largely exceeding the ELVs are needed-to potentially induce adverse effects. Considering MRI operators, in case of whole-body static field exposure, at levels above $8 \mathrm{~T}$ nerve stimulation and involuntary muscle contraction can be induced especially during fast movements, and only in case of exposures largely above the ELVs more relevant effects, as changes in blood flow in limbs and/or heart rate, can possibly appear. Especially in case of the latter effects, the knowledge is limited, mainly related to few studies and case reports; and the relevance in terms of human's health is currently not adequately understood.

On the contrary, for indirect effects, and in particular for interference problems, it is not possible to consider the ELVs protective for all the "workers at particular risk", as those wearing active medical devices: this specific scenario will be discussed in detail in the following sub-section.

In general, the HS of MRI operators (as well as for other occupational risks) has to be based on the results of the assessment of the risks arising from EMF exposure at the workplace, that is an obligation of the employer [153].

At the moment no authoritative and/or shared guidelines exist on the specific contents of the HS of MRI operators, including possible biological tests or other monitoring or screening methods for preventive purposes according to the ethic code of the International Commission of Occupational Health [154], even if 
some general principle can be found e.g. in the previously mentioned "Non-binding guide" [68], in particular in the chapter 11 "Risks, symptoms and health surveillance".

According to the 35/2013 EU Directive, HS is also needed when "if any undesired or unexpected health effect is reported by a worker, or in any event where exposure above the ELVs is detected, the employer shall ensure that appropriate medical examinations or individual health surveillance is provided to the worker(s) concerned, in accordance with national law and practice". Such "medical examination or individual health surveillance" should be considered something different from the "routine" HS. The approach recommended is that such medical examinations should be performed by occupational physicians with adequate expertise in the prevention of the EMF-related occupational risk, integrating the examination with specific medical consultation(s) if needed and, in case, laboratory tests and/or diagnostic exams defined on an individual clinical evaluation of the subject. This point seems particularly relevant for MRI operators as, according to the scientific literature, at least some subjective symptoms are not exceptional in these workers $[7,35,129$, 130, 155-157]. Furthermore, according to the article 10 of the Directive, under "specific circumstances", derogations for the use of MRI, also in case of exposures exceeding the exposure limit values (ELVs), are permitted. These "specific circumstances" include an application of all the technical and/or organizational preventive measures taking into account the characteristics of the work activity, the recognition of a justified need for exceeding the ELVs and a demonstration provided by the employer showing that workers have still a valid protection against the EMF related adverse health effects and safety risks, respecting the safe-use instructions of the manufacturer and with defined procedures to be followed, in case, to ensure the respect of the Medical Devices Directive (93/42/EEC) [158].

The possible occurrence of sensory symptoms during MRI exposure should be investigated during the HS of MRI operators, and their possible causes should be evaluated, as well as their reported occurrence in relation to the MRI activities, and in particular in case of rapid movements of the operators close to the MRI scanner. As anticipated, in various studies the occurrence of different subjective symptoms has been observed in MRI operators $[7,35,129,130,155-157]$. These symptoms, usually resulting from self-administrated questionnaire studies, are mainly non-specific, e.g. migraine, asthenia, memory loss, and their occurrence may be confounded by other occupational and non-occupational risk factors (e.g. distress, concomitant diseases and related therapies, etc). On the other hand, in some recent studies a group of five more specific 
symptoms have been proposed as "core symptoms": vertigo, nausea, head ringing, magnetophosphenes and metallic taste, and may be related to a direct sensory effect, based on induction of currents in the body, of the static magnetic field exposure of the operators, in particular if they perform (rapid) movements within the field. A systematic collection of the occurrence of these core symptoms and of their evolution overtime should be usefully considered for the HS of MRI operators $[35,130]$.

A last point to be highlighted here is that the subjective symptoms, even if considered rapidly reversible and without clinical consequences, may increase the risk of work-related accidents, e.g. the fall of the operator. Another aspect, scarcely or not addressed so far, is the possibility that the occurrence of these symptoms may interfere with attention, possibly inducing the risk of procedural errors. Interestingly, the topic of accidents, and in particular of car accidents and nearly-missed car accidents after work, was recently addressed in two Dutch studies, one performed in a cohort of workers engaged in a MRI scanners production facility [9] and the other in a group of MRI operators [11]: the results suggest an increased risk of accidents and of selfreported nearly-missed, possibly related to attention interference, supporting the opportunity of further research on this topic.

\subsection{Workers at particular risk}

Another relevant aspect to be considered in the HS of MRI operators is the possible presence of "workers at particular risk": to date, there is no exhaustive catalogue of conditions inducing a particular risk. The EU Directive 35/2013 does not provide a full list of these conditions, but at least a (partial) definition is given in Article 5, where it is recommended to carry out "individual risks assessments, in particular in respect of workers who have declared the use of active or passive implanted medical devices, such as cardiac pacemakers, or the use of medical devices worn on the body, such as insulin pumps, or in respect of pregnant workers who have informed their employer of their condition" [17]. A more detailed list of conditions determining a particular sensitivity to the risk is presented in the first volume of the previously mentioned "Non-binding guide" [68], in the Table 3.1. In this case, the workers at particular risk are categorized in 4 groups: 
workers wearing active implanted medical devices: e.g. cardiac pacemakers, cardiac defibrillators, cochlear implants, brainstem implants, inner ear prostheses, neurostimulators, retinal encoders, implanted drug infusion pumps;

$\checkmark$ workers wearing passive implanted medical devices containing metal: e.g. artificial joints, pins, plates, screws, surgical clips, aneurism clips, stents, heart valve prostheses, annuloplasty rings, metallic contraceptive implants, and cases of AIMD;

workers wearing body-worn medical devices: e.g. external hormone infusion pumps;

pregnant workers.

In all the cases considering an implanted or body-worn device, the condition of particular susceptibility to the risk is due to a possible indirect effect of the EMF exposure. More specifically, for all the "active" devices, the main indirect effects are the possibility of interferences with the electrical activity of the device. Furthermore, for all devices, active and passive, in case of presence of metallic parts other possible indirect effects to be considered, as a mechanical effect possibly inducing a dislocation of the device, or of part(s) of it, and the induction of contact currents, possibly causing heating or triggering inflammation of the surrounding tissue. These two latter mechanisms are of concerns mainly in case of high exposures. Currently, most/all the prosthesis, vascular clips and stents, contraceptive devices and others are nonmagnetic. Furthermore, it has to be noted that EMF indirect interactions are possible also with non-medical, and non-active, body inclusions, e.g. metal splinters as a consequence of accidents, or body piercings or, in the past, also metallic pigments used for tattoos.

The main problem is related to the risk of interference of EMF with active devices, possible also in the case of relatively low EMF exposure, below the ELVs defined in the 2013 EU Directive and, in some cases, possibly even below the limits recommended for the general public. Accordingly, this issue should deserve a particular consideration for the protection of MRI operators and for their health surveillance. Pacemakers and ICDs are the most studied active implanted medical devices with regard to their possible interference with EMF: alterations of the sensing functions of the devices, with possible consequences as inhibition of the stimulation or inappropriate stimulations, and in the worst cases a permanent damage of the device requiring a resetting or a substitution have been reported. A further important consideration is that, in many occasions, minor interferences with AIMD are completely reversible and clinically silent, so that the workers do not 
perceive any malfunction and the problems are discovered only with a periodical check of the devices, that are able to register their activities, when interference episodes could be detected and analyzed.

Nevertheless, fortunately, considering the scientific literature available to date, reports of in vivo malfunctions of AIMD in EMF-exposed workers are very few (especially considering the diffusion of these devices), and the large part of data showing relevant interference problems of AIMD within static magnetic fields produced by MRI scanners, are based on studies on mannequin models. In such studies interference were often observed for older devices with an unipolar configuration, while recent bipolar devices are less, or not, involved. Furthermore, an increasing number of AIMD is considered "safe" for patients who need a MRI examination (MRI-conditional), and in some studies no long-term clinically significant adverse events were reported in patients with pacemakers or ICDs, including devices that were not considered MRIconditional [159]. In the case of occupational exposures, AIMDs, including the MRI-conditional devices, are currently not considered adequately safe for the occupational activity of MRI operators, and accordingly, in case the risk evaluation identifies a significant EMF exposure, as e.g. when a worker needs to access the Controlled Access Area, AIMDs are considered a specific contraindication for this working activity; similar considerations can also be applied to most passive devices with ferromagnetic components (see also Section 3.1).

Regarding the other conditions of particular risk for the workers exposed to EMF, the European Directive 35/2013 explicitly identifies pregnancy. In this case, the types of EMF induced effects to be considered are the direct ones, even if it should be noted that scientific data showing an increased sensitivity of pregnant women to static or low frequency EMF are scant, and inconclusive. Some available data suggest the possibility of an increased susceptibility of the fetus to the thermal effect related to RF exposures, and a possible association between maternal cell-phone use during pregnancy and shorter pregnancy duration/ increased risk of preterm birth has been found in a recent study [160], but such effects should be confirmed, and, in any case, RF exposures are usually not significant for MRI operators. On the other hand it has to be considered that the Council Directive 92/85/EEC [161] includes exposure to non-ionizing radiation among the activities liable to involve a specific risk for pregnant women: considering these premises, a compliance with the ELVs established by the European Directive 2013/35 possibly cannot be considered sufficiently protective for pregnant workers, and it would be much advisable to refer to lower exposure limits, such as 
those set for the general population according to the European Recommendation 1999/519/EC [144] and more recently updated for some EMF frequency bands by the ICNIRP. As most usually the exposure limits for the general population are (largely) exceeded in MRI activities, at least in Europe, working with MRI should be avoided in pregnant operators or, in any case, the engagement in MRI activities during pregnancy is contraindicated.

Excluding the aforementioned problems of subjects with AIMD and other medical devices, and the pregnancy condition, little and not very sound scientific evidence currently exists on other conditions determining a particular risk related to occupational EMF exposure of MRI operators.

\subsection{Discussion}

Considering direct and indirect effects of the occupational exposure to EMF and the problem of the "workers at particular risk", the HS of MRI operators should be based on the active systematic seeking of symptoms (and signs) possibly related to EMF-associated effects [162]. The occupational physicians should look for and follow not only the occurrence but also the possible evolutions/changes of symptoms, possibly in relation to occupational exposure.

Specific attention in the course of HS of MRI operators should be devoted to the workers "at particular risk". To some extent, this latter point is still open as, except for the subjects with implanted medical devices and pregnant worker, knowledge on other specific conditions inducing a "particular risk" (if any), and on the exposure levels inducing a risk, are far from being adequately defined to date [68]. Regarding the criteria and the contents of an "adequate" HS of EMF-exposure in MRI operators, up until now, there is no agreement, though a specific objective should be, at least, the (early) detection of EMF-related adverse effects, such as the stimulation of muscles, nerves or sensory organs (including temporary annoyance or effects on cognition), and thermal effects [162]. As the "core symptoms" have been frequently reported in MRI operators, at least the occurrence of these symptoms, and the monitoring of their evolution, seem appropriate for the HS; specific questionnaires could be useful tools for this purpose. In the case of strict compliance with the current ELVs, these effects are usually prevented, but an exceeding of the ELV is possible (or, rather, is probable) during MRI activities. Furthermore, ELVs may not be adequately protective in the case of "workers at particular risk": for this reason, the identification of these workers is an essential component 
of an appropriate HS program. Regarding possible tests and other monitoring procedures, to date, no specific laboratory tests or other medical investigations have been demonstrated as effective for an appropriate HS of MRI operators.

The results of such HS in EMF-exposed MRI operators are particularly important to identify, and adequately protect, the workers with conditions possibly inducing a particular risk. In this respect, important aspects of the EU Directive 2013/35/EU, not sufficiently considered up now, are the requirement that the results of HS "shall be preserved in a suitable form that allows them to be consulted at a later date" (Art. 8), and the possibility for the Commission to acquire "the appropriate information that it receives from Member States," to keep the European Parliament and the Council informed about any new evidence on the possible health effects related to EMF occupational exposure [17].

\section{Ultra-high field MRI}

As regards the ultra-high field scanners, such as $7 \mathrm{~T}$ or superior, actually there are no specific concerns for occupational safety, although currently more than 70 ultra-high field scanners are operated around the world [163]. The EU Directive prescribes, as the upper limit for static magnetic field exposure, $8 \mathrm{~T}$ for controlled working conditions. For all work procedures that do not involve reaching into the bore of the magnet, it is straightforward to demonstrate compliance.

The latest generations of ultra-high field are actively shielded: this causes a very high spatial gradient of the SMF close to the bore [30]. Considering as exposure metrics the maximum variation of the SMF on every 3s-interval, for comparison with the ICNIRP basic restriction aimed at preventing vertigo effects [50], and two weighted-peak indices for "sensory" and "health" effects to prevent stimulation effects $[17,50]$, a lot of worker procedures in a $7 \mathrm{~T}$ scanner have proved to be non-compliant with the regulations [69]. This shows that compliance with EU Directive limits for SMF is not sufficient to guarantee compliance with reference levels imposed by ICNIRP for movement-induced stimulation effects.

Staff should hence avoid placing their head into the bore whenever possible and should move slowly in the proximity of the bore. Otherwise, workers should be mindful of potential transient effects which 
could persist for some time after exposure, compromising some activities, such as driving, immediately afterward the exposure $[164,165]$. In specific studies regarding occupational exposure in ultra-high field scanners, the most frequently reported sensation is dizziness/vertigo that can potentially be stimulated by the SMF and by the movement within the field [166, 167].

Studies in the literature reported that exceedances of the reference level for the time-varying magnetic field at 3 and $7 \mathrm{~T}$ were almost similar [124].

Analysis of the max SMF experienced by workers of $7 \mathrm{~T}$ scanner revealed a large variability between participants, even though the paths followed for the approaching the bore were identical for all the participants [124]. This fact suggests again the importance of performing personal exposure assessment.

\section{Fig 2 here}

\section{Conclusions}

Safeguarding the health of workers in MR is a very complex issue and still partially to be explored. In this review, the current knowledge about occupational exposure to EMF in a magnetic resonance environment has been summarized, mainly dealing with specific aspects not always appropriately addressed, such as the regulations, the literature studies on biological effects, and health surveillance. Some useful tips have been deduced in support of better safeguarding of MRI operators (Fig. 2).

Several critical points are raised by the analysis of the literature. First of all, we have to consider that MRI staff includes different operators, engaged in a range of rather different tasks (i.e. patient positioning and removal, system verification test, calibration, hardware tests, ...). This implies a very large variability in the dosimetric parameters with complications to perform a rigorous exposure assessment, health surveillance, and epidemiological studies about chronic exposure [3, 33]. For this reason, one of the first suggestions regards an exposure categorization for the different professions working with MRI equipment, that could be 
based, for example, on the nature of the fields they are exposed to, the time that they are exposed, and the procedures that they perform. Some tools generally used to assess exposure to potential health hazards in occupational epidemiological studies, such as the job-exposure matrix, could aid $[9,168,169]$.

Regarding the exposure assessment, it emerged that there are no standardized procedures to assess the MRI occupational exposure. If on the one hand there is evidence for the development of innovative approaches, on the other side better transparency, reliability, and efficiency are needed to increase the reproducibility of these methods. The exposure assessment certainly requires detailed knowledge of the spatial distribution of fields in each specific MRI environment with an understanding of how staff move, something that will be strongly dependent on the tasks to be completed. It is with this in mind that numerical modelling techniques can be useful, both to evaluate compliance with imposed limits and to set up rigorous epidemiological studies. Moreover, our careful analysis showed that there are only a few studies related to the risk assessment of MRI workers with passive and active biomedical implants. In this respect, the numerical calculation approaches will certainly aid in the future.

Regarding the biological effects arising from the complex exposure to EMFs in MRI workers, the most reported effects that are associated with a longer duration of working in the MRI department are dizziness, vertigo, and nausea. The evidence on the possible effects of exposure below the thresholds of acute effects is not sufficient for a proper health risk assessment. Only a few epidemiological studies have been carried out and they address the effects of SMF exposure in terms of cancer risk.

Regarding effects on DNA integrity and in germ cells, only sporadic cases found an effect and it is not clear to which component of the MRI exposure the effect can be ascribed. From this, it follows the importance of having a detailed description of the MRI exposure to make the experiments repeatable in different laboratories.

From the point of view of health surveillance of MRI operators, it has come to light that no authoritative and/or shared guidelines exist as well as no exhaustive catalog of conditions inducing a "particular risk" has available. Precise guidelines should be made available in the future for a systematic seeking of symptoms (occurrence and evolutions/changes) and precise identification of "workers at particular risk".

The current international system of regulations is essentially based on the ICNIRP guidelines for limiting exposure to EMFs, which set specific limits for different frequency range (static $(0 \mathrm{~Hz})$, TvMF below $1 \mathrm{~Hz}$, 
low-frequency $(1 \mathrm{~Hz}$ to $100 \mathrm{kHz}), \mathrm{RF}(100 \mathrm{kHz}$ to $300 \mathrm{GHz})$. The ICNIRP guidelines refer both to the general public and occupational exposure to EMFs without specifying the nature of the source from which they are produced. These guidelines do not address specific device standards for limiting EMF emission.

A key point to consider is that the ICNIRP guidelines, and all the resulting regulations, are based on all known short-term direct biophysical effects and indirect effects caused by EMF, but do not address suggested long-term effects because no sufficient evidence has been found at the moment. Another important point regards the EU Directive that sets a non-discretionary derogation on the installation, testing, use, development, maintenance of, or research related to the use of MRI, which allows exposure to exceed the safety limits providing certain conditions are satisfied. This implies that the employer has to undertake a series of specific actions, such as a rigorous risk assessment and efficient worker training and information. Worker education should involve all the aspects relative to occupational exposure to EMF and in particular: the results of the exposure assessment with the measurement or calculation of the levels of exposure, how to detect and report transient symptoms, sensations, and adverse health effects related to the exposure of EMF, conditions in which the workers are entitled to health surveillance, especially for workers at particular risk, and finally best practices to minimise risks.

From a practical point of view, concerning the movements of the staff inside the MRI environment, it would be appropriate to identify a series of procedures, eventually to converge on at least national guidelines, to avoid the instant overcoming of the action limits imposed by the international safety regulations. With particular regard to the SMF spatial gradients, MRI workers may be exposed to induced electric fields which cause sensory effects also of relevant importance, such as vertigo, nausea, head ringing, magnetophosphenes, as well as a general feeling of discomfort. This could happen for instance during an emergency, when the worker, alerted by critical clinical conditions of the patient, moves too quickly within an area that, in normal conditions, has no particular risks. For this reason, it would be appropriate to indicate on the floor, with a high visibility color, the area with the higher value of spatial gradient, in which the worker movement speed has to be kept below a threshold value (typically $1 \mathrm{~m} / \mathrm{s}$ ) to avoid the instant overcoming of the action limits. This high-risk area should be identified for each specific MRI environment, considering the worst-case scenario with the maximum value of induced electric field given by the estimation of the magnetic field flow 
through a standard human thorax moving with uniform rectilinear motion at high speed and a standard human head rotating with a high angular speed.

Another critical point to consider is related to ultra-high field MRI scanners, already highly widespread, especially in research centres. As they are primarily used for research purposes, these scanners are not subject to the same regulations as clinical scanners. The issue of occupational exposure to ultra-high EMFs MRI is therefore far to be fully defined for the safeguarding of the health of researchers working in such environments. In this regard, future studies on specific risk assessment for such MRI environments are desirable.

At the end of this review, we can assert that the issue of exposure to the EMFs of MRI workers involves different professional categories with multidisciplinary skills. All the treated fundamental aspects should be interconnected so that the results of scientific research are reflected in the implementation of regulations and the creation of best practices, to transfer the new knowledge to everyday working practice.

\section{Figure and Table captions}

Fig. 1 Timeline of main regulations about general public and occupational exposure to electromagnetic fields

Fig. 2 Key points and main indications for specific aspects of occupational safety in MRI

Table 1 Comparison between the different national standards present in Europe regarding MRI

\section{References}

1. McRobbie DW, Moore EA, Graves MJ, Prince MR (2017) MRI from Picture to Proton. Cambridge University Press, Cambridge

2. OECD (2017) Health at a Glance 2017: OECD Indicators. https://doi.org/10.1787/health_glance-2017-en

3. Hansson Mild K, Hand J, Hietanen M, et al (2013) Exposure classification of MRI workers in epidemiological studies. Bioelectromagnetics 34:81-4. https://doi.org/10.1002/bem.21728 
4. McRobbie DW (2020) Essentials of MRI safety. Wiley-Blackwell, Hoboken, New Jersey, US

5. Van Nierop LE, Slottje P, Kingma H, Kromhout H (2013) MRI-related static magnetic stray fields and postural body sway: A double-blind randomized crossover study. Magn Reson Med 70:232-240. https://doi.org/10.1002/mrm.24454

6. van Nierop LE, Slottje P, Zandvoort M Van, Kromhout H (2015) Simultaneous Exposure to MRI-Related Static and Magnetic Fields Affects Neurocognitive Performance: A DoubleBlind Randomized Crossover Study. Magn Reson Med 849:840-849. https://doi.org/10.1002/mrm.25443

7. Zanotti G, Ligabue G, Korpinen L, Gobba F (2016) Subjective symptoms in Magnetic Resonance Imaging operators: prevalence, short-term evolution and possible related factors. Med del Lav 107:263-270

8. Walker M, Fultz A, Davies C, Brockopp D (2020) Symptoms Experienced by MR Technologists Exposed to Static Magnetic Fields. Radiol Technol 91:316-323

9. Bongers S, Slottje P, Portengen L, Kromhout H (2016) Exposure to static magnetic fields and risk of accidents among a cohort of workers from a medical imaging device manufacturing facility. Magn Reson Med 75:2165-2174. https://doi.org/10.1002/mrm.25768

10. Bongers S, Slottje P, Kromhout H (2018) Development of hypertension after long-term exposure to static magnetic fields among workers from a magnetic resonance imaging device manufacturing facility. Environ Res 164:565-573. https://doi.org/10.1016/j.envres.2018.03.008

11. Huss A, Schaap K, Kromhout H (2017) MRI-related magnetic field exposures and risk of commuting accidents - A cross-sectional survey among Dutch imaging technicians. Environ Res 156:613-618. https://doi.org/10.1016/j.envres.2017.04.022

12. Huss A, Ozdemir E, Schaap K, Kromhout H (2021) Occupational exposure to MRI-related magnetic stray fields and sleep quality among MRI - Technicians - A cross-sectional study in the Netherlands. Int J Hyg Environ Health 231:113636. https://doi.org/10.1016/j.ijheh.2020.113636

13. ICNIRP (1998) Guidelines for limiting exposure to time-varying electric, magnetic, and electromagnetic fields (up to 300 GHz). Health Phys 74:494-522. 
https://doi.org/10.1097/HP.0b013e3181aff9db

14. ICNIRP (2009) Guidelines on Limits of Exposure To Static Magnetic Fields. Health Phys 96:504-514. https://doi.org/10.1097/01.HP.0000343164.27920.4a

15. IEEE (2006) Standard for Safety Levels with Respect to Human Exposure to Radio Frequency Electromagnetic Fields, $3 \mathrm{kHz}$ to $300 \mathrm{GHz}$. Institute of Electrical and Electronics Engineers

16. IEEE (2002) Standard for Safety Levels with Respect to Human Exposure to Electromagnetic Fields, 0-3 kHz. Institute of Electrical and Electonics Engineers

17. European Parliament and Council of the European Union (2013) Directive 2013/35/EC on the minimum health and safety requirements regarding the exposure of workers to the risks arising from physical agents (electromagnetic fields). Off J Eur Union L179/1-21

18. Kemper VG, De Martino F, Emmerling TC, et al (2018) High resolution data analysis strategies for mesoscale human functional MRI at 7 and 9.4T. Neuroimage 164:48-58. https://doi.org/10.1016/j.neuroimage.2017.03.058

19. Kagadis GC, Ford NL, Karnabatidis DN, et al (2016) Handbook of Small Animal Imaging : Preclinical Imaging, Therapy, and Applications. CRC Press, Taylor and Francis Group, Cleveland, Ohio, US

20. Hartwig V, Romeo S, Zeni O (2018) Occupational exposure to electromagnetic fields in magnetic resonance environment: basic aspects and review of exposure assessment approaches. Med Biol Eng Comput 56:531-545. https://doi.org/10.1007/s11517-017-1779-7

21. Simmons A, Hakansson K, Sammet S, et al (2016) Magnetic resonance safety. Abdom Radiol 41:17-29. https://doi.org/10.1007/s00261-016-0680-4

22. Panych LP, Madore B (2018) The physics of MRI safety. J Magn Reson Imaging 47:28-43. https://doi.org/10.1002/jmri.25761

23. Frankel J, Wilén J, Hansson Mild K (2018) Assessing Exposures to Magnetic Resonance Imaging's Complex Mixture of Magnetic Fields for In Vivo, In Vitro, and Epidemiologic Studies of Health Effects for Staff and Patients. Front Public Heal 6:. https://doi.org/10.3389/fpubh.2018.00066

24. Hartwig V (2016) Risk of Magnetic Resonance: The Safety-Biological Effects. In: Saba L (ed) 
Image Principles, Neck, and the Brain. CRC Press, pp 191-212

25. Hartwig V, Giovannetti G, Vanello N, et al (2009) Biological effects and safety in magnetic resonance imaging: a review. Int J Env Res Public Heal 6:1778-1798. https://doi.org/10.3390/ijerph6061778

26. Stafford RJ (2020) The Physics of Magnetic Resonance Imaging Safety. Magn Reson Imaging Clin N Am 28:517-536. https://doi.org/10.1016/j.mric.2020.08.002

27. SCENIHR (2015) Potential health effects of exposure to electromagnetic fields (EMF). SCENIHR Rep 1-288. https://doi.org/10.2772/75635

28. Betta G, Capriglione D, Pasquino N (2012) Experimental investigation on workers' exposure to electromagnetic fields in proximity of magnetic resonance imaging systems. Measurement 45:199-206. https://doi.org/10.1016/j.measurement.2011.03.001

29. Karpowicz J, Hietanen M, Gryz K (2007) Occupational risk from static magnetic fields of MRI scanners. Environmentalist 27:533-538. https://doi.org/10.1007/s10669-007-9064-1

30. Hartwig V, Biagini C, De Marchi D, et al (2019) The Procedure for Quantitative Characterization and Analysis of Magnetic Fields in Magnetic Resonance Sites for Protection of Workers: A Pilot Study. Ann Work Expo Heal 63:1-9. https://doi.org/10.1093/annweh/wxz002

31. Hartwig V, Vanello N, Giovannetti G, et al (2011) A novel tool for estimation of magnetic resonance occupational exposure to spatially varying magnetic fields. MAGMA 24:323-330. https://doi.org/10.1007/s10334-011-0279-2

32. Hartwig V, Vanello N, Giovannetti G, et al (2014) Estimation of Occupational Exposure to Static Magnetic Fields Due To Usual Movements in Magnetic Resonance Units. Concepts Magn Reson Part B Magn Reson Eng 44:75-81. https://doi.org/10.1002/cmr.b.21270

33. Hartwig V, Biagini C, Marchi D De, et al (2020) Analysis, comparison and representation of occupational exposure to a static magnetic field in a 3-T MRI site. Int J Occup Saf Ergon 0:110. https://doi.org/10.1080/10803548.2020.1738114

34. Gurrera D, Gallias KK, Spanò M, et al (2019) Moving across the static magnetic field of a 1.5 T MRI scanner: Analysing compliance with Directive 2013/35/EU. Phys Medica 57:238- 
244. https://doi.org/10.1016/j.ejmp.2018.11.004

35. Schaap K, Christopher-de Vries Y, Mason CK, et al (2014) Occupational exposure of healthcare and research staff to static magnetic stray fields from 1.5-7 Tesla MRI scanners is associated with reporting of transient symptoms. Occup Environ Med 71:423-429. https://doi.org/10.1136/oemed-2013-101890

36. Schaap K, Christopher-De Vries Y, Slottje P, Kromhout H (2013) Inventory of MRI applications and workers exposed to MRI- related electromagnetic fields in the Netherlands. Eur J Radiol 82:2279-2285. https://doi.org/10.1016/j.ejrad.2013.07.023

37. Te.Si.A. (2017) Mafiss-Te.Si.A. TEcnologie e SInergie Applicate Srl. http://tesiasrl.it/ma-fi-ss-dispositivo-rilevatore-campi-magnetici-statici/. Accessed 11 Dec 2018

38. Crozier S, Wilson SJ, Gregg I (2011) US7936168B2 Magnetic field dosimeter

39. Fuentes MA, Trakic A, Wilson SJ, Crozier S (2008) Analysis and measurements of magnetic field exposures for healthcare workers in selected MR environments. IEEE Trans Biomed Eng 55:1355-1364. https://doi.org/10.1109/TBME.2007.913410

40. Batistatou E, Molter A, Kromhout H, et al (2016) Personal exposure to static and time-varying magnetic fields during MRI procedures in clinical practice in the UK. Occup Environ Med 73:779-786. https://doi.org/10.1136/oemed-2015-103194

41. Delmas A, Weber N, Piffre J, et al (2017) MRI "exposimetry": How to analyze, compare and represent worker exposure to static magnetic field? Radiat Prot Dosimetry 177:415-423. https://doi.org/10.1093/rpd/ncx060

42. Tecnorad (2017) Talete-Tecnorad Personal Dosimetry Service. http://www.tecnorad.it/campimagnetici.php. Accessed 11 Dec 2018

43. Hartwig V, Virgili G, Ferrante Vero LF, et al (2018) Towards a Personalised and Interactive Assessment of Occupational Exposure To Magnetic Field During Daily Routine in Magnetic Resonance. Radiat Prot Dosimetry 182:1-9. https://doi.org/10.1093/rpd/ncy114

44. Hartwig V, Ferrante Vero LFLF, Virgili G, et al (2019) Device for the assessment of occupational exposure to time-varying magnetic field due to movement in magnetic resonance environments. Electron Lett 55:579-581. https://doi.org/10.1049/el.2019.0275 
45. Schaap K, Christopher-De Vries Y, Cambron-Goulet É, Kromhout H (2016) Work-related factors associated with occupational exposure to static magnetic stray fields from MRI scanners. Magn Reson Med 75:2141-2155. https://doi.org/10.1002/mrm.25720

46. Acri G, Testagrossa B, Vermiglio G (2015) Personal Time-Varying Magnetic Fields Evaluation During Activities in MRI Sites. In: IFMBE Proceedings. pp 741-744

47. Filice S, Rossi R, Crisi G (2019) Assessment of Movement-Induced Time-Varying Magnetic Fields Exposure in Magnetic Resonance Imaging By a Commercial Portable Magnetometer. Radiat Prot Dosimetry 2014:1-5. https://doi.org/10.1093/rpd/ncz016

48. Sannino A, Romeo S, Scarfì MR, et al (2017) Exposure Assessment and Biomonitoring of Workers in Magnetic Resonance Environment: An Exploratory Study. Front Public Heal 5:344. https://doi.org/10.3389/fpubh.2017.00344

49. Farrag SI (2015) Numerical Simulation of the Induced Currents in Occupational Workers Induced by Body-Motion around Different MRI Fields. Int J Adv Res Comput Sci Softw Eng 5:51-55

50. ICNIRP (2014) Guidelines for limiting exposure to electric fields induced by and by timevarying magnetic fields below 1 Hz. Health Phys 106:418-425. https://doi.org/10.1097/HP.0b013e31829e5580

51. Cobos Sánchez C, Glover P, Power H, Bowtell R (2012) Calculation of the electric field resulting from human body rotation in a magnetic field. Phys Med Biol 57:4739-53. https://doi.org/10.1088/0031-9155/57/15/4739

52. Zilberti L, Bottauscio O, Chiampi M (2015) Motion-Induced Fields in Magnetic Resonance Imaging: Are the Dielectric Currents Really Negligible? IEEE Magn Lett 6:1-4. https://doi.org/10.1109/LMAG.2015.2429641

53. Zilberti L, Bottauscio O, Chiampi M (2016) Assessment of exposure to MRI motion-induced fields based on the International Commission on Non-Ionizing Radiation Protection (ICNIRP) guidelines. Magn Reson Med 76:1291-1300. https://doi.org/10.1002/mrm.26031

54. Laakso I, Kännälä S, Jokela K (2013) Computational dosimetry of induced electric fields during realistic movements in the vicinity of a 3 T MRI scanner. Phys Med Biol 58:2625-40. 
https://doi.org/10.1088/0031-9155/58/8/2625

55. Chiampi M, Zilberti L (2011) Induction of Electric Field in Human Bodies Moving Near MRI: An Efficient BEM Computational Procedure. IEEE Trans Biomed Eng 58:2787-2793

56. Andreuccetti D, Contessa GM, Falsaperla R, et al (2013) Weighted-peak assessment of occupational exposure due to MRI gradient fields and movements in a nonhomogeneous static magnetic field. Med Phys 40:011910. https://doi.org/10.1118/1.4771933

57. Bonutti F, Tecchio M, Maieron M, et al (2016) Measurement of the weighted peak level for occupational exposure to gradient magnetic fields for 1.5 and 3 tesla MRI body scanners. Radiat Prot Dosimetry 168:358-364. https://doi.org/10.1093/rpd/ncv308

58. Di Liberto R, Andreuccetti D, Comelli M (2021) Assessment of the Exposure to Gradient Magnetic Fields Generated by MRI Tomographs: Measurement Method, Verification of Limits and Clearance Areas through a Web-Based Platform. Int J Environ Res Public Heal Artic 18:3475

59. Wang H, Trakic A, Liu F, Crozier S (2008) Numerical field evaluation of healthcare workers when bending towards high-field MRI magnets. Magn Reson Med 59:410-422. https://doi.org/10.1002/mrm.21441

60. Crozier S, Wang H, Trakic A, Liu F (2007) Exposure of workers to pulsed gradients in MRI. J Magn Reson Imaging 26:1236-1254. https://doi.org/10.1002/jmri.21162

61. Riches SF, Collins DJ, Scuffham JW, Leach MO (2007) EU Directive 2004/40: Field measurements of a $1.5 \mathrm{~T}$ clinical $\mathrm{MR}$ scanner. $\mathrm{Br} \quad \mathrm{J}$ Radiol 80:483-487. https://doi.org/10.1259/bjr/69843752

62. Gourzoulidis G, Karabetsos E, Skamnakis N, et al (2015) Occupational Electromagnetic Fields exposure in Magnetic Resonance Imaging systems - Preliminary results for the RF harmonic content. Phys Medica 31:757-762. https://doi.org/10.1016/j.ejmp.2015.03.006

63. Stralka JP, Bottomley PA (2007) A prototype RF dosimeter for independent measurement of the average specific absorption rate (SAR) during MRI. J Magn Reson Imaging 26:1296-1302. https://doi.org/10.1002/jmri.21141

64. Yee KS (1966) Numerical Solution of Initial Boundary Value Problems Involving Maxwell's 
Equations in Isotropic Media. IEEE Trans Antennas Propag 14:302-307

65. Hartwig V, Giovannetti G, Vanello N, et al (2010) Numerical Calculation of Peak-to-Average Specific Absorption Rate on Different Human Thorax Models for Magnetic Resonance Safety Considerations. Appl Magn Reson 38:337-348

66. Christ A, Kainz W, Hahn EG, et al (2010) The Virtual Family--development of surface-based anatomical models of two adults and two children for dosimetric simulations. Phys Med Biol 55:N23-38. https://doi.org/10.1088/0031-9155/55/2/N01

67. ICNIRP (2010) Guidelines for limiting exposure to time-varying electric and magnetic fields (1 Hz to 100 kHz). Health Phys 99:818-36. https://doi.org/10.1097/HP.0b013e3181f06c86

68. European Commission (2015) Non-binding guide to good practice for implementing Directive 2013/35/EC Electromagnetic Fields Volume 1: Practical Guide

69. Andreuccetti D, Biagi L, Burriesci G, et al (2017) Occupational exposure in MR facilities due to movements in the static magnetic field. Med Phys 44:5988-5996. https://doi.org/10.1002/mp.12537

70. Zilberti L, Bottauscio O, Chiampi M (2016) A Potential-Based Formulation for MotionInduced Electric Fields in MRI. IEEE Trans Magn 52:. https://doi.org/10.1109/TMAG.2015.2474748

71. ICNIRP (2003) Guidance on determining compliance of exposure to pulsed fields and complex non-sinusoidal waveforms below $100 \mathrm{kHz}$ with ICNIRP guidelines. Health Phys 84:383-387

72. European Commission (2015) Non-binding guide to good practice for implementing Directive 2013/35/EC Volume 2: Case Studies

73. European Committee for Electrotechnical Standardization (2016) EN 50527-1:2016, Procedure for the Assessment of the Exposure to Electromagnetic Fields of Workers Bearing Active Implantable Medical Devices_-Part 1: General

74. European Committee for Electrotechnical Standardization (2016) EN 50527-2-1:2016, Procedure for the Assessment of the Exposure to Electromagnetic Fields of Workers Bearing Active Implantable Medical Devices-Part 2-1: Specific Assessment for Workers with Cardiac Pacemakers 
75. European Committee for Electrotechnical Standardization (2018) EN 50527-2-2:2018, Procedure for the Assessment of the Exposure to Electromagnetic Fields ofWorkers Bearing Active Implantable Medical Devices-Part 2-2: Specific Assessment for Workers with Cardioverter Defibrillators (ICDs)

76. European Parliament and of the Council (2017) Regulations (EU) 2017/745 on medical devices. Off J Eur Union L 117:1-228

77. Mattei E, Calcagnini G, Censi F, et al (2019) Workers with active implantable medical devices exposed to emf: In vitro test for the risk assessment. Environ - MDPI 6:1-13. https://doi.org/10.3390/environments6110119

78. Mattei E, Censi F, Calcagnini G, et al (2016) Pacemaker and ICD oversensing induced by movements near the MRI scanner bore. Med Phys 43:6621-6631. https://doi.org/10.1118/1.4967856

79. Mattei E, Censi F, Triventi M, et al (2015) Wrong Detection of Ventricular Fibrillation in an Implantable Cardioverter Defibrillator Caused by the Movement Near the MRI Scanner Bore. In: Conf Proc IEEE Eng Med Biol Soc. pp 7200-3

80. Shellock FG, Woods TO, Crues J V. (2009) MR Labeling Information for Implants and Devices: $\quad$ Explanation $\quad$ of $\quad$ Terminology. $\quad$ Radiology https://doi.org/10.1148/RADIOL.2531091030

81. Zeng Q, Wang Q, Zheng J, et al (2018) Evaluation of MRI RF electromagnetic field induced heating near leads of cochlear implants. Phys Med Biol 63:. https://doi.org/10.1088/1361$6560 /$ aacbf2

82. Srinivasan R, So CW, Amin N, et al (2019) A review of the safety of MRI in cochlear implant patients with retained magnets. Clin Radiol 74:972.e9-972.e16. https://doi.org/10.1016/j.crad.2019.06.011

83. Winter L, Seifert F, Zilberti L, et al (2020) MRI-Related Heating of Implants and Devices: A Review. J Magn Reson Imaging 1-20. https://doi.org/10.1002/jmri.27194

84. Arduino A, Zanovello U, Hand J, et al (2021) Heating of hip joint implants in MRI: The combined effect of RF and switched-gradient fields. Magn Reson Med 1-16. 
https://doi.org/10.1002/mrm.28666

85. Fujimoto K, Angelone LM, Lucano E, et al (2018) Radio-frequency safety assessment of stents in blood vessels during magnetic resonance imaging. Front Physiol 9:1-10. https://doi.org/10.3389/fphys.2018.01439

86. Gobba F, Bianchi N, Verga P, et al (2012) Menometrorrhagia in magnetic resonance imaging operators with copper intrauterine contraceptive devices (iuds): A case report. Int J Occup Med Environ Health 25:97-102. https://doi.org/10.2478/s13382-012-0005-y

87. Huss A, Schaap K, Kromhout H (2018) A survey on abnormal uterine bleeding among radiographers with frequent MRI exposure using intrauterine contraceptive devices. Magn Reson Med 79:1083-1089. https://doi.org/10.1002/mrm.26707

88. Zradziński P (2015) Difficulties in applying numerical simulations to an evaluation of occupational hazards caused by electromagnetic fields. Int J Occup Saf Ergon 21:213-220. https://doi.org/10.1080/10803548.2015.1028233

89. Burgio E, Piscitelli P, Migliore L (2018) Ionizing Radiation and Human Health: Reviewing Models of Exposure and Mechanisms of Cellular Damage. An Epigenetic Perspective. Int J Environ Res Public Health 15:. https://doi.org/10.3390/ijerph15091971

90. Schaap K, Christopher-De Vries Y, Crozier S, et al (2014) Exposure to static and time-varying magnetic fields from working in the static magnetic stray fields of MRI scanners: A comprehensive survey in the Netherlands. Ann Occup Hyg 58:1094-1110. https://doi.org/10.1093/annhyg/meu057

91. Karpowicz J, Gryz K (2006) Health risk assessment of occupational exposure to a magnetic field from magnetic resonance imaging devices. Int J Occup Saf Ergon 12:155-167. https://doi.org/10.1080/10803548.2006.11076679

92. Vogt FM, Ladd ME, Hunold P, et al (2004) Increased Time Rate of Change of Gradient Fields: Effect on Peripheral Nerve Stimulation at Clinical MR Imaging. Radiology 233:548-554. https://doi.org/10.1148/radiol.2332030428

93. Davids M, Guérin B, vom Endt A, et al (2019) Prediction of peripheral nerve stimulation thresholds of MRI gradient coils using coupled electromagnetic and neurodynamic simulations. 
Magn Reson Med 81:686-701. https://doi.org/10.1002/mrm.27382

94. Staebler P (2017) Human Exposure to Electromagnetic Fields: From Extremely Low Frequency (ELF) to Radiofrequency. John Wiley \& Sons, Inc., Hoboken, NJ, USA

95. Brix G, Kolem H, Nitz WR, et al (2008) Basics of Magnetic Resonance Imaging and Magnetic Resonance Spectroscopy. In: Magnetic Resonance Tomography. Springer Berlin Heidelberg, Berlin, Heidelberg, pp 3-167

96. ICNIRP (2004) ICNIRP Statement On Medical Magnetic Resonance (MR) Procedures: Protection Of Patients. Health Phys 87:197-216

97. Dewhirst MW, Viglianti BL, Lora-Michiels M, et al (2003) Basic principles of thermal dosimetry and thermal thresholds for tissue damage from hyperthermia. Int J Hyperth 19:267294. https://doi.org/10.1080/0265673031000119006

98. van den Brink JS (2019) Thermal Effects Associated with RF Exposures in Diagnostic MRI: Overview of Existing and Emerging Concepts of Protection. Concepts Magn Reson Part B 2019:1-17. https://doi.org/10.1155/2019/9618680

99. Mattei E, Lucano E, Censi F, et al (2016) High dielectric material in MRI: Numerical assessment of the reduction of the induced local power on implanted cardiac leads. Proc Annu Int Conf IEEE Eng Med Biol Soc EMBS 2016-Octob:2361-2364. https://doi.org/10.1109/EMBC.2016.7591204

100. Shellock FG (2014) Reference Manual for Magnetic Resonance Safety, Implants, and Devices: 2014 Edition. Biomedical Research Publishing Group, Playa Del Rey, CA

101. Schaefers G, Melzer A (2011) Devices and Materials in MRI. In: Springer Handbook of Medical Technology. Springer Berlin Heidelberg, Berlin, Heidelberg, pp 503-521

102. Schlamann M, Voigt MA, Maderwald S, et al (2010) Exposure to high-field MRI does not affect cognitive function. J Magn Reson Imaging 31:1061-1066. https://doi.org/10.1002/jmri.22065

103. Mollerlokken OJ, Moen BE, Baste V, et al (2012) No effects of MRI scan on male reproduction $\quad$ hormones. $\quad$ Reprod $\quad$ Toxicol 34:133-139. https://doi.org/10.1016/j.reprotox.2012.04.003 
104. R. Thulborn K, C. Atkinson I, H. Pliskin N, Jamil S (2019) Safety of 9.4 Tesla for Neuroimaging of Healthy and For-Cause Volunteers. J Radiol Clin Imaging 02:23-33. https://doi.org/10.26502/jrci.2644-2809011

105. van Nierop LE, Slottje P, van Zandvoort MJE, et al (2012) Effects of magnetic stray fields from a 7 Tesla MRI scanner on neurocognition: a double-blind randomised crossover study. Occup Environ Med 69:759-766. https://doi.org/10.1136/oemed-2011-100468

106. Grant A, Metzger GJ, Van de Moortele PF, et al (2020) 10.5 T MRI static field effects on human cognitive, vestibular, and physiological function. Magn Reson Imaging 73:163-176. https://doi.org/10.1016/j.mri.2020.08.004

107. Simi S, Ballardin M, Casella M, et al (2008) Is the genotoxic effect of magnetic resonance negligible? Low persistence of micronucleus frequency in lymphocytes of individuals after cardiac scan. Mutat Res - Fundam Mol Mech Mutagen 645:39-43. https://doi.org/10.1016/j.mrfmmm.2008.08.011

108. Fiechter M, Stehli J, Fuchs TA, et al (2013) Impact of cardiac magnetic resonance imaging on human lymphocyte DNA integrity. Eur Heart J 34:2340-2345. https://doi.org/10.1093/eurheartj/eht184

109. Lancellotti P, Nchimi A, Delierneux C, et al (2015) Biological Effects of Cardiac Magnetic Resonance on Human Blood Cells. Circ Cardiovasc Imaging 8:1-9. https://doi.org/10.1161/CIRCIMAGING.115.003697

110. Brand M, Ellmann S, Sommer M, et al (2015) Influence of cardiac MR imaging on DNA double-strand breaks in human blood lymphocytes. Radiology 277:406-412. https://doi.org/10.1148/radiol.2015150555

111. Fatahi M, Reddig A, Vijayalaxmi, et al (2016) DNA double-strand breaks and micronuclei in human blood lymphocytes after repeated whole body exposures to 7T Magnetic Resonance Imaging. Neuroimage 133:288-293. https://doi.org/10.1016/j.neuroimage.2016.03.023

112. Reddig A, Fatahi M, Roggenbuck D, et al (2017) Impact of in Vivo high-field-strength and ultra-high-field- strength $\mathrm{mr}$ imaging on DNA double-strand-break formation in human lymphocytes. Radiology 282:782-789. https://doi.org/10.1148/radiol.2016160794 
113. Fasshauer M, Krüwel T, Zapf A, et al (2018) Absence of DNA double-strand breaks in human peripheral blood mononuclear cells after 3 Tesla magnetic resonance imaging assessed by $\gamma$ H2AX flow cytometry. Eur Radiol 28:1149-1156. https://doi.org/10.1007/s00330-017-5056-9

114. Suntharalingam S, Kraff O (2018) Healthy Volunteers : Relationship to Formation of DNA Double-Strand Breaks. Radiology 288:529-535

115. Jalali AH, Mozdarani H, Ghanaati H (2020) The genotoxic effects of contrast enhanced abdominopelvic 3-tesla magnetic resonance imaging on human circulating leucocytes. Eur J Radiol 129:109037. https://doi.org/10.1016/j.ejrad.2020.109037

116. Critchley WR, Reid A, Morris J, et al (2018) The effect of $1.5 \mathrm{~T}$ cardiac magnetic resonance on human circulating leucocytes. Eur Heart J 39:305-312. https://doi.org/10.1093/eurheartj/ehx646

117. Rostamzadeh A, Anjamrooz SH, Rezaie MJ, et al (2019) Biological effects of magnetic resonance imaging on testis histology and seminiferous tubules morphometry. Oman Med J 34:544-552. https://doi.org/10.5001/omj.2019.98

118. Lee JW, Kim MS, Kim YJ, et al (2011) Genotoxic effects of 3T magnetic resonance imaging in cultured human lymphocytes. Bioelectromagnetics 32:535-542. https://doi.org/10.1002/bem.20664

119. Szerencsi Á, Kubinyi G, Váliczkó É, et al (2013) DNA integrity of human leukocytes after magnetic resonance imaging. Int J Radiat Biol 89:870-876. https://doi.org/10.3109/09553002.2013.804962

120. Reddig A, Fatahi M, Friebe B, et al (2015) Analysis of DNA double-strand breaks and cytotoxicity after 7 tesla magnetic resonance imaging of isolated human lymphocytes. PLoS One 10:1-14. https://doi.org/10.1371/journal.pone.0132702

121. Friebe B, Godenschweger F, Fatahi M, et al (2018) The potential toxic impact of different gadolinium-based contrast agents combined with 7-T MRI on isolated human lymphocytes. Eur Radiol Exp 2:1-9. https://doi.org/10.1186/s41747-018-0069-y

122. Vijayalaxmi, Fatahi M, Speck O (2015) Magnetic resonance imaging (MRI): A review of genetic damage investigations. Mutat Res - Rev Mutat Res 764:51-63. 
https://doi.org/10.1016/j.mrrev.2015.02.002

123. Hill MA, O’Neill P, McKenna WG (2016) Comments on potential health effects of MRIinduced DNA lesions: quality is more important to consider than quantity. Eur Heart J Cardiovasc Imaging 17:1230-1238. https://doi.org/10.1093/ehjci/jew163

124. Fatahi M, Karpowicz J, Gryz K, et al (2017) Evaluation of exposure to (ultra) high static magnetic fields during activities around human MRI scanners. Magn Reson Mater Physics, Biol Med 30:255-264. https://doi.org/10.1007/s10334-016-0602-z

125. Mild KH, Lundström R, Wilén J (2019) Non-ionizing radiation in swedish health careexposure and safety aspects. Int $\mathrm{J}$ Environ Res Public Health 16:. https://doi.org/10.3390/ijerph16071186

126. Wilén J, Olsrud J, Frankel J, Hansson Mild K (2020) Valid Exposure Protocols Needed in Magnetic Resonance Imaging Genotoxic Research. Bioelectromagnetics 41:247-257. https://doi.org/10.1002/bem.22257

127. Feychting M (2005) Health effects of static magnetic fields - A review of the epidemiological $\begin{array}{lllll}\text { evidence. } & \text { Prog } & \text { Biophys 241-246. }\end{array}$ https://doi.org/10.1016/j.pbiomolbio.2004.08.007

128. Heinrich A, Szostek A, Nees F, et al (2011) Effects of static magnetic fields on cognition, vital signs, and sensory perception: a meta-analysis. J Magn Reson Imaging 34:758-63. https://doi.org/10.1002/jmri.22720

129. De Vocht F, Batistatou E, Mölter A, et al (2015) Transient health symptoms of MRI staff working with 1.5 and 3.0 Tesla scanners in the UK. Eur Radiol 25:2718-2726. https://doi.org/10.1007/s00330-015-3629-z

130. Schaap K, Portengen L, Kromhout H (2016) Exposure to MRI-related magnetic fields and vertigo in MRI workers. Occup Environ Med 73:161-6

131. Tian X, Wang D, Feng S, et al (2019) Effects of 3.5-23.0 T static magnetic fields on mice: A safety study. Neuroimage 199:273-280. https://doi.org/10.1016/j.neuroimage.2019.05.070

132. Wang S, Luo J, Lv H, et al (2019) Safety of exposure to high static magnetic fields (2 T-12 T): a study on mice. Eur Radiol 29:6029-6037. https://doi.org/10.1007/s00330-019-06256-y 
133. Miyakoshi J (2006) The review of cellular effects of a static magnetic field. Sci Technol Adv Mater 7:305-307. https://doi.org/10.1016/j.stam.2006.01.004

134. Ghodbane S, Lahbib A, Sakly M, Abdelmelek H (2013) Bioeffects of Static Magnetic Fields : Oxidative Stress, Genotoxic Effects, and Cancer Studies. Biomed Res Int 2013:. https://doi.org/10.1155/2013/602987

135. Romeo S, Sannino A, Scarfî MR, et al (2016) Lack of effects on key cellular parameters of MRC-5 human lung fibroblasts exposed to $370 \mathrm{mT}$ static magnetic field. Sci Rep 6:19398. https://doi.org/10.1038/srep19398

136. Tian X, Wang D, Zha M, et al (2018) Magnetic field direction differentially impacts the growth of different cell types. Electromagn Biol Med 37:114-125. https://doi.org/10.1080/15368378.2018.1458627

137. Zhang L, Ji X, Yang X, Zhang X (2017) Cell type- and density-dependent effect of $1 \mathrm{~T}$ static magnetic field on cell proliferation. Oncotarget 8:13126-13141. https://doi.org/10.18632/oncotarget.14480

138. Bodewein L, Schmiedchen K, Dechent D, et al (2019) Systematic review on the biological effects of electric, magnetic and electromagnetic fields in the intermediate frequency range (300 Hz to $1 \mathrm{MHz}$ ). Environ Res 171:247-259. https://doi.org/10.1016/j.envres.2019.01.015

139. De Vocht F, Liket L, De Vocht A, et al (2007) Exposure to alternating electromagnetic fields and effects on the visual and visuomotor systems. $\mathrm{Br} \quad \mathrm{J}$ Radiol 80:822-828. https://doi.org/10.1259/bjr/22263979

140. Glover PM, Eldeghaidy S, Mistry TR, Gowland PA (2007) Measurement of visual evoked potential during and after periods of pulsed magnetic field exposure. J Magn Reson Imaging 26:1353-1356. https://doi.org/10.1002/jmri.21155

141. International Electrotechnical Commission (IEC) (2007) IEC 62464-1:Magnetic resonance equipment for medical imaging - Part 1: Determination of essential image quality parameters

142. International Electrotechnical Commission (IEC) (2013) IEC-60601-2-33-1:Amendment 1 Medical electrical equipment - Part 2-33: Particular requirements for the basic safety and essential performance of magnetic resonance equipment for medical diagnosis 
143. European Committee for Electrotechnical Standardization (CENELEC) (2008) Basic standard on measurement and calculation procedures for human exposure to electric, magnetic and electromagnetic fields $(0 \mathrm{~Hz}-300 \mathrm{GHz})$

144. Council of the European Communities (1999) CELEX1, 1999/519/EC: Council Recommendation of 12 July 1999 on the limitation of exposure of the general public to electromagnetic fields ( $0 \mathrm{~Hz}$ to $300 \mathrm{GHz}$ ). Publications Office of the European Union

145. Council of the European Communities (1989) Council Directive 89/391/EEC of 12 June 1989 on the introduction of measures to encourage improvements in the safety and health of workers at work. Off J Eur Communities 183:8. https://doi.org/2004R0726 - v.7 of 05.06.2013

146. ICNIRP (2020) ICNIRP Guidelines for limiting exposure to electromagnetic fields $(100 \mathrm{kHz}$ TO 300 GHz). Health Phys 118:483-524. https://doi.org/10.1097/HP.0000000000001210

147. Presidenza della Repubblica Italiana (2016) Decreto Legislativo 1 agosto 2016, n. 159. GU $192: 1-15$

148. Ministero della Salute (2018) Decreto Ministeriale 10 agosto 2018. GU 236:1-21

149. American College of Radiology Committee on MR Safety (2020) ACR Manual on MR Safety

150. US Food and Drug Administration (FDA) Manufacturer and user facility device experience database (MAUDE): file formats for FOI releasable data. www.fda.gov/cdrh/maude.html

151. International Labour Organization (1998) Technical and ethical guidelines for workers' health surveillance. Occupational Safety and Health Series No. 72, Geneva

152. Hocking B, Gobba F (2011) Medical aspects of overexposures to electromagnetic fields. J Heal Saf Environ 27:185-195

153. Gobba F, Korpinen L (2018) What health surveillance of EMF exposed workers? Occup Environ Med 75:2018

154. International Commission on Occupational Health (2012) International Code of Ethics for occupational health professionals. $1-19$

155. Wilén J, Vocht F De (2011) Health complaints among nurses working near MRI scanners - A descriptive pilot study. Eur J Radiol 80:510-513. https://doi.org/10.1016/j.ejrad.2010.09.021

156. Friebe B, Wollrab A, Thormann M, et al (2015) Sensory perceptions of individuals exposed to 
the static field of a 7T MRI: A controlled blinded study. J Magn Reson Imaging 41:1675-1681. https://doi.org/10.1002/jmri.24748

157. Zanotti G, Ligabue G, Gobba F (2015) Subjective symptoms and their evolution in a small group of magnetic resonance imaging (MRI) operators recently engaged. Electromagn Biol Med 34:262-264. https://doi.org/10.3109/15368378.2015.1076442

158. The Council Of European Comminuties (1993) Council Directive 93/42/EEC of 14 June 1993 concerning medical device. 1-43

159. Nazarian S, Hansford R, Rahsepar AA, et al (2017) Safety of magnetic resonance imaging in patients with cardiac devices. N Engl J Med 377:2555-2564. https://doi.org/10.1056/NEJMoa1604267

160. Tsarna E, Reedijk M, Birks LE, et al (2019) Associations of Maternal Cell-Phone Use During Pregnancy With Pregnancy Duration and Fetal Growth in 4 Birth Cohorts. Am J Epidemiol 188:1270-1280. https://doi.org/10.1093/aje/kwz092

161. European Union (1992) Council Directive 92/85/EEC of 19 October 1992 on the introduction of measures to encourage improvements in the safety and health at work of pregnant workers and workers who have recently given birth or are breastfeeding. Off J Eur Communities L $348: 1-7$

162. Modenese A, Gobba F (2020) Occupational Exposure to Non-Ionizing radiation. Main effects and criteria for health surveillance of workers according to the European Directives. Proc 2020 IEEE Int Conf Environ Electr Eng 2020 IEEE Ind Commer Power Syst Eur EEEIC / I CPS Eur 2020. https://doi.org/10.1109/EEEIC/ICPSEurope49358.2020.9160831

163. Friebe B (2020) Editorial for "Subjectively reported Effects Experienced in an Actively Shielded 7T MR: A Large-Scale Study.” J Magn Reson Imaging 52:1277-1278. https://doi.org/10.1002/jmri.27157

164. Theysohn JM, Kraff O, Eilers K, et al (2014) Vestibular effects of a 7 tesla MRI examination compared to $1.5 \mathrm{~T}$ and $0 \mathrm{~T}$ in healthy volunteers. PLoS One 9:3-10. https://doi.org/10.1371/journal.pone.0092104

165. Fagan AJ, Bitz AK, Björkman-Burtscher IM, et al (2021) 7T MR Safety. J Magn Reson 
Imaging 53:333-346. https://doi.org/10.1002/jmri.27319

166. Hansson B, Markenroth Bloch K, Owman T, et al (2020) Subjectively Reported Effects Experienced in an Actively Shielded 7T MRI: A Large-Scale Study. J Magn Reson Imaging 52:1265-1276. https://doi.org/10.1002/jmri.27139

167. Fatahi M, Demenescu LR, Speck O (2016) Subjective perception of safety in healthy individuals working with 7 T MRI scanners: a retrospective multicenter survey. Magn Reson Mater Phy 29:379-387. https://doi.org/10.1007/s10334-016-0527-6

168. Bongers S, Christopher Y, Engels H, et al (2013) Retrospective Assessment of Exposure to Static Magnetic Fields During Production and Development of Magnetic Resonance Imaging Systems. Ann Occup Hyg 58:85-102. https://doi.org/10.1093/annhyg/met049

169. Migault L, Bowman JD, Kromhout H, et al (2019) Development of a job-exposure matrix for assessment of occupational exposure to high-frequency electromagnetic fields $(3 \mathrm{kHz}-300$ GHz). Ann Work Expo Heal 63:1013-1028. https://doi.org/10.1093/annweh/wxz067

Authors' biographies

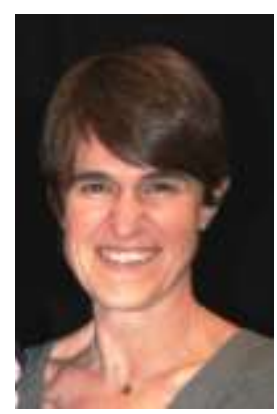


Valentina Hartwig received the Degree in Electronic Engineering, with a curriculum in Biomedical Engineering, in 2003 from the University of Pisa and PhD degree in Automatics, Robotics and Bioengineering at Engineering faculty of Pisa, in 2010. Since 2012 she is with IFC-CNR as Researcher working on biological effects and safety in Magnetic Resonance Imaging. She is author of many papers published in peer-reviewed journals, conference proceedings, and book chapters, mainly on modelling and analysis of the interaction between magnetic fields and biological tissues and on exposure assessment methods in MRI environment.

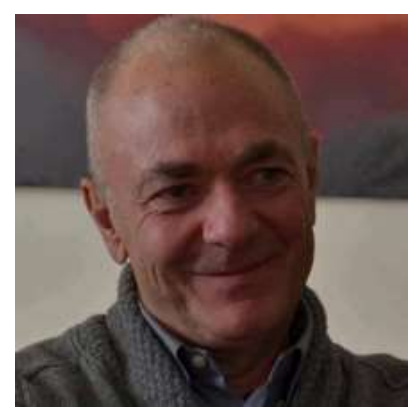

Giorgio Virgili began his professional career in 1980, as a specialist for ionizing radiations instrumentation, at the Italian Ministry of Defence; from 1989 to 2020 he was with WEST Systems as responsible for the design of instrumentation and protocols for the chemical-physical characterization of the environment. He currently operates as an independent consultant.

\section{Federica Mattei na}

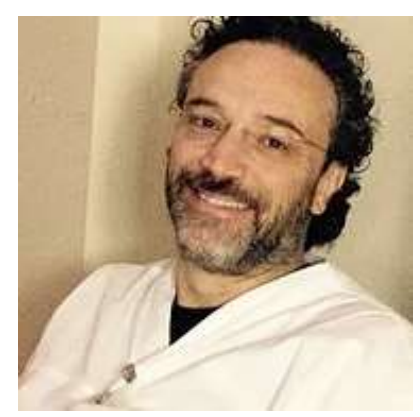

Cristiano Biagini received the degree in Physics in 1995, the PhD in theoretical solid state physics in 1999, a second degree as a radiographer/technologist in 2009, and a post-graduation Medical Physics degree of the Università Cattolica in Rome in June 2016. He worked on protocols and sequences otpimization of MRI 
scanners. He's currently working on Body Oncologic advanced applications, like DWI, IVIM and DCEMRI, particularly on their technical foundations and on the use of MRI in radiotherapy treatment planning.

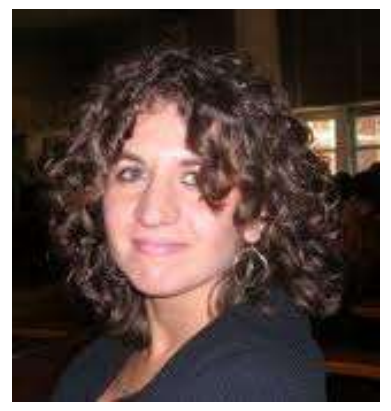

Stefania Romeo received the degree in Biomedical Eng. in 2008 and the PhD in Electronic Eng. in 2012. She is with IREA-CNR as Researcher working on the technical and engineering aspects of bioelectromagnetic interactions.

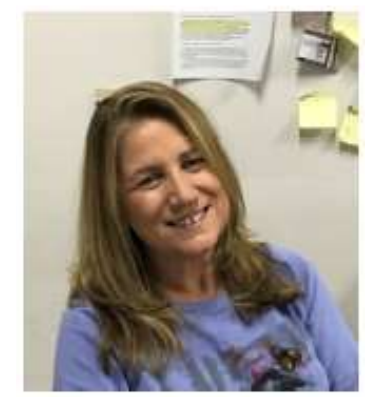

Olga Zeni, biologist, $\mathrm{PhD}$, is involved in the study of interactions between electromagnetic fields and mammalian cells. She has experience in the revision of scientific literature on this topic in the framework of EU, WHO and IEEE working groups.

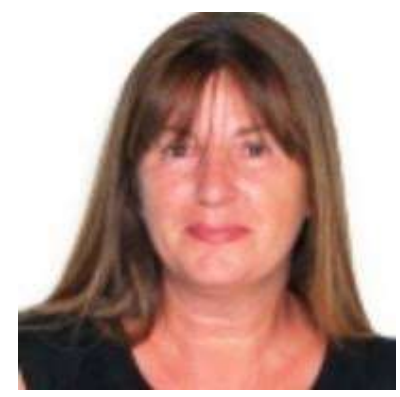

Maria Rosaria Scarfî, biologist, has long lasting experience in study of interactions between electromagnetic fields and mammalian cells. She has experience in the revision of scientific literature on this topic in the framework of the E.U., WHO, and Swedish Radiation Safety Authority working groups. 


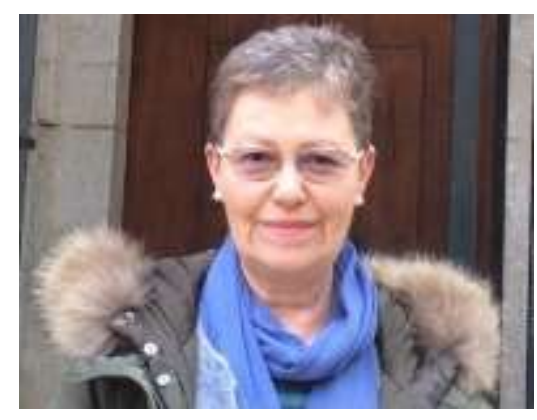

Rita Massa received the Physics degree (summa cum laude) and Ph.D. degree in Electronic and Computer Science from the University of Naples Federico II, Naples, Italy. Since 1992, she is at the University of Naples Federico II and, actually, as Professor of Electromagnetic Fields at the Department of Physics. She has been the chief of the Microwave Interaction Division (MIND), responsible for the Biophysics Laboratory and actually she is the chief of Non Ionizing Radiation Laboratory (DP). She is currently the Director of the "Interuniversity Center for the Study of Interactions between Electromagnetic Fields and Biosystems (ICEmB)". Her research interests are is in the framework of the interactions of electromagnetic fields and materials, dealing with the biological effects of Electromagnetic Fields, electromagnetic dosimetry/exposure assessment, therapeutic and industrial applications of Electromagnetic Fields, and nondestructive testing of materials. She was and is currently involved in research projects, in cooperation with small, medium and/or great enterprises, for which she played and is currently playing the role of scientific coordinator/WP Leader. She has coauthored more than 200 papers published in peer-reviewed journals, proceedings, and conference papers.

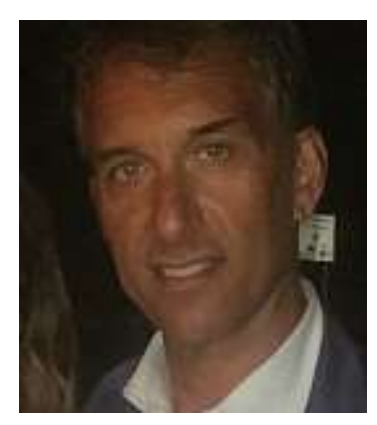

Francesco Campanella graduated in Physics in 1991 at the University of Rome "La Sapienza". In 1997 he was a researcher at ISPESL and in 1998 he obtained the title of qualified expert for second degree radiation 
protection. In 2010 he moved to the National Institute for Insurance against Accidents at Work (INAIL). Since 2016 he is a member of the Technical Support Section to the National Health Service regarding radiation of INAIL. He is author of many guidelines and good practices in the areas of competence.

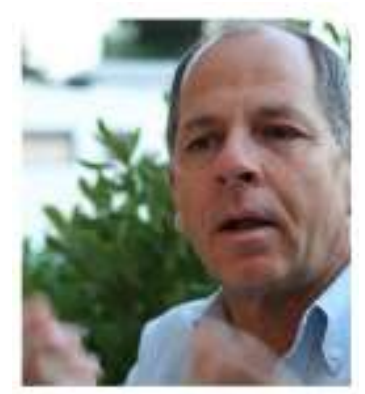

Luigi Landini received the degree in Physics from Pisa University, Italy, in 1974. From 1975 to 1981 he was research fellow firstly at the Institute of Clinical Physiology in Pisa, after at the Engineering Faculty in Florence and finally at the Interfaculty Center 'E. Piaggio' of the Engineering Faculty in Pisa. In 1981 he joined the Engineering Faculty of Pisa as Assistant Professor. Since 1998 he is Professor of Biomedical Engineering at the Engineering Faculty of Pisa and he teaches biomedical signal and image processing at Biomedical Engineering degree at the University of Pisa. He is scientific coordinator of medical imaging projects at the Fondazione Gabriele Monasterio in Pisa and he is engaged in research on biomedical signal and image processing. He is author of more than 400 publications on modeling and analysis of biomedical signal and images.

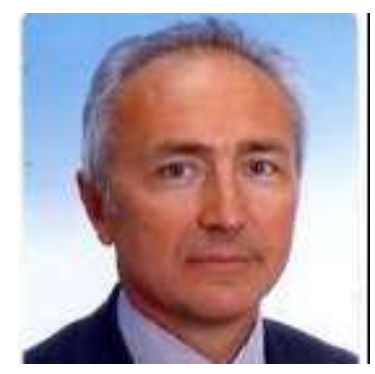

Fabriziomaria Gobba (M.D.) is Full Professor of Occupational Medicine at the University of Modena and Reggio Emilia, Director/Coordinator of the Postgraduate Specialization School of Occupational Medicine of the University of Modena and Reggio Emilia, Chair of the Scientific Committee "Radiation and Work" of the International Commission of Occupational Health (ICOH) up to 2018, Vice President of the Italian 
Association of Medical Radioprotection (AIRM) and Member of the National Board of the of the Italian Society of Occupational Medicine (SIML), Member of the Board of Directors and of the Scientific Council of the National Research Center Interuniversity on Interactions between Electromagnetic Fields and Biosystems (ICEmB). - Author of several papers in scientific journals and Proceedings/Book of abstracts of national and international conferences in the field of the risk related to the occupational exposure to NonIonizing Radiation (NIR) and prevention in workers.

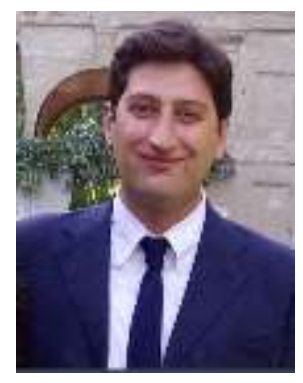

Alberto Modenese is an occupational physician, Post-Doc Researcher at the Chair of Occupational Medicine of the University of Modena and Reggio Emilia (Modena, Italy). He got a post-graduate specialization degree in Occupational Medicine in 2014, and a Ph.D. in "Clinical and Experimental Medicine" in 2017. Is Secretary of the Scientific Committee "Radiation and Work" of the International Commission of Occupational Health $(\mathrm{ICOH})$. His main area of research is the studying of preventive measures, exposure evaluation methods and health effects related to Non-Ionizing Radiation (NIR) exposure in workers.

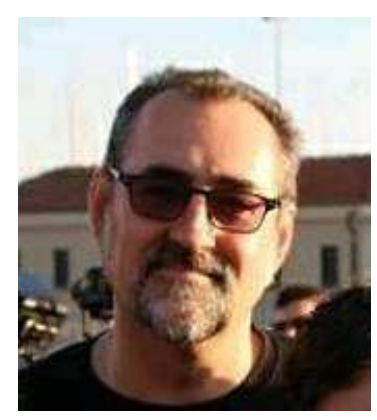

Giulio Giovannetti received the Master's degree in electronic engineering, the Master's degree in biomedical engineering, and the Ph.D. degree in automatics, robotics, and bioengineering from the University of Pisa, Italy, in 2000, 2004, and 2008, respectively. Since 2009, he is a Researcher at the C.N.R. 
Institute of Clinical Physiology in Pisa. His research interests include electromagnetic field simulations and measurements in magnetic resonance environment, radiofrequency coil design and simulation for magnetic resonance imaging and spectroscopy. 